\title{
Le site paléontologique du Grand Morier (Pont-Boutard, Indre-et-Loire, France): contexte géologique et détail biostratigraphique des formations cénozoïques à partir des assemblages de vertébrés fossiles
}

\author{
Cyril GAGNAISON \\ Institut Polytechnique UniLaSalle Beauvais, Département Géosciences, Equipe B2R, \\ 19 rue Pierre Waguet - boîte postale 30313, F-60026 Beauvais cedex (France) \\ cyril.gagnaison@unilasalle-beauvais.fr
}

Publié le 30 juin 2017

MOTS CLÉS

Vertébrés,

Cénozoïque,

Miocene,

France,

Touraine,

biostratigraphie, tectonique,

assemblage faunique,

remaniement

taphonomie.

Gagnaison C. 2017. - Le site paléontologique du Grand Morier (Pont-Boutard, Indre-et-Loire, France): contexte géologique et détail biostratigraphique des formations cénozoïques à partir des assemblages de vertébrés fossiles. Geodiversitas 39 (2): 251-271. https://doi.org/10.5252/g2017n2a5

\section{RÉSUMÉ}

Un site paléontologique du Sud-Ouest du Bassin parisien est décrit: l'ancienne falunière du Grand Morier. Il se situe à l'ouest de la ville de Langeais et au sud du hameau de Pont-Boutard (Indreet-Loire, France). Le contexte géologique du Grand Morier est complexe. Toutes les formations sédimentaires cénozoïques locales sont représentées sur le site: les formations continentales étagées depuis le Paléogène moyen jusqu'au Miocène, les faluns marins du Miocène et les formations continentales de la fin du Néogène. Les phénomènes de tectonique locale (phases pyrénéenne et alpine) ont contraint et canalisé la sédimentation cénozoïque dans une structure en graben. De nombreux fossiles de vertébrés cénozoïques ont été trouvés dans le site paléontologique du Grand Morier. La consultation de 450 fossiles de vertébrés conservés dans différentes collections a permis de réaliser une liste faunique composée de 102 taxons. Les données géologiques et taphonomiques ont permis de reconstituer des assemblages de fossiles de même âge et de même état de conservation. Six ensembles biostratigraphiques bien distincts ressortent : deux faunes continentales en place de l'Éocène (MP12 à MP20) et Oligocène-Agénien supérieur (MP24 à MN2), une faune marine en place - ou faiblement remaniée - dans les faluns miocènes (Burdigalien supérieur à Tortonien) et trois faunes remaniées (Crétacé supérieur au Miocène) dans les faluns miocènes. Ces assemblages de fossiles de vertébrés recadrent et précisent la stratigraphie du Cénozoïque local. 


\begin{abstract}
The paleontological site of Le Grand Morier (Pont-Boutard, Indre-et-Loire, France): geological context and vertebrate biostratigraphy of the Cenozoic formations.

A paleontological site of the Paris Basin is described here: Le Grand Morier quarry. This site is located West of the Langeais city and South of the hamlet of Pont-Boutard (Indre-et-Loire, France). The geological context of Le Grand Morier is complex. The site comprises all local late Cenozoic sedimentary formations cropping out locally: the Paleogene to lower Orleanian continental formations, the Miocene marine shelly sands and the Upper Neogene continental formations. Local tectonic phenomena (Pyrenean and Alpine phases) created a graben structure, which has focused most of the Cenozoic sedimentation. Many fossils of Cenozoic vertebrates have been found in the paleontological site of Le Grand Morier. For the purpose of this study, 450 fossils of vertebrates (102 taxa) have been studied in various paleontological collections. The geological and taphonomic contexts enabled the recognition of fossil clusters based on their hypothesized age and preservation state. The paleontological study reveals six distinct groups: two in situ continental faunas Eocene (MP12 to MP20) and Oligocene-Early Miocene (MP24 to MN2) in age, an in situ or slightly reworked Miocene marine fauna (upper Burdigalian to Tortonian) and three clusters of reworked fossils (upper Cretaceous to Miocene) in the Miocene shelly sands. These vertebrate fossils clusters improve the resolution of the Cenozoic stratigraphic framework of the area.
\end{abstract}

\title{
ABRIDGED ENGLISH VERSION
}

The paleontological site of Le Grand Morier is located in the heart of the Ligerian Basin, 40 kilometers West from the city of Tours (Temey 1996). This fossiliferous site corresponds to an old quarry where the Miocene shelly sand was exploited (Fig. 1). The most important geological and paleontological studies of Le Grand Morier are those of Lecointre (1930, 1947), Alcaydé (1975) and Alcaydé et al. (1976) for the geological surveys (geological mapping, stratigraphy and structural studies) and of Buge (1948) and Ginsburg (2001) for their work on the fossils (bryozoans and Miocene land mammals respectively).

This article presents original data on the geological context and the stratigraphy of the Cenozoic sedimentary series of Le Grand Morier site (Fig. 2). Cenozoic outcrops are numerous and diversified in Anjou-Touraine (Lecointre 1947). Looking at the regional geological maps is enough to realize that the Cretaceous is covered by continental Paleogene formations and marine Neogene formations (Alcaydé \& Rasplus 1971; Alcaydé 1975). To our knowledge, Le Grand Morier is the only known site in Anjou-Touraine with exposures of all Cenozoic formations (albeit small outcrops for some of them), from the Paleocene-Eocene "perrons de Touraine" to the Late Miocene to Pliocene gravel sands (Fig. 4). This site is exceptional because vertebrate fossils have been found in each outcropping formation. In addition, this paleontological site is located into a local tectonic structure: the PontBoutard graben (Fig. 3). Integrating the structural and stratigraphic clearly shows that the Cenozoic sedimentation is a syntectonic sedimentation constrained and channeled by the structurally-active Pont-Boutard graben. Specifically, the establishment of the continental formations (fluviatile and lacustrine deposits) during the middle Cenozoic (Eocene to earliest Miocene) was made possible by the formation of a basin controlled by two faults activated by the Pyrenan orogeny (Fig. 2). The Alpine orogeny (Miocene) accentuated the deepening of the Pont-Boutard graben, safeguarding a major part of the Cenozoic sedimentary series (Paleocene to Miocene), but also redirecting the prograding marine sediments from the basin of Savigné-sur-Lathan/Noyant-sous-le-Lude towards the basin of Paulmy in Southern Touraine.

By compiling all the geological (stratigraphy, sedimentology, and taphonomy) and paleontological data, it is possible to consider six successive assemblages of vertebrate faunas (102 taxa), reworked or not. The assemblage no. 1 contains one tooth of Plagiolophus cf. annectens (Owen, 1847) (Fig. 7); found in the Middle to Late Eocene lacustrine green clay (MP12 to MP20). The assemblage no. 2 contains four land mammals either Oligocene (Ronzotherium cf. romani: MP24-29) or Early Miocene in age (Agenian-Aquitanian): Phoberocyon hispanicus Ginsburg \& Morales, 1998, Protaceratherium minutum (Cuvier, 1822-1824), Paratapirus intermedius (Filhol, 1885) (Fig. 7). The fossils have been found in the lacustrine limestone underlying the Miocene marine shelly sand. It is an important new input for the Cenozoic biostratigraphy of the Anjou-Touraine area. This lacustrine limestone known as "le calcaire lacustre de Touraine", has always been dated as Late Eocene to Oligocene (Alcaydé 1975; Yvard 1976). The presence in our site of land mammals similar to the fossils observed in the Early Miocene locality of Selles-sur-Cher (Loir-et-Cher, France, Ginsburg \& Hugueney 1980; Antoine et al. 2000) together with a typical Oligocene rhinocerotid enables us to consider that geological formation may range from the late early Oligocene to the Agenian, MP24 to MN2 (Bruijn et al. 1992; Aguilar et al. 1997). The assemblage no. 3 is exclusively composed of well-known Miocene marine vertebrates (fishes and mammals): Isurus desori, Cosmopolitodus hastalis, Otodus megalodon, Carcharias (Odontaspis) acutissima 
ou Carcharias (Odontaspis) cuspidata, Carcharias sp., Carcharhinus priscus, Galeocerdo aduncus, Negaprion kraussi, Hemipristis serra, Notorhynchus primigenius, Dasyatis sp., Myliobatidae indet., Aetobatus arcuatus, Pristis aquitanicus, Sparus cinctus, Sparus aurata, Diplodus jomnitanus, Sphyraena olisiponensis, Labrodon pavimentatum, Tetrodon lecointrae, Trigonodon jugleri, Metaxytherium sp., Cetacea indet. and Orycterocetus crocodilinus. Numerous publications have already presented this typical faunal list of the Ligerian basin (Mornand 1978; Ginsburg \& Mornand 1986) the age of which ranges covers most of the Miocene epoch: from the late Burdigalian to the Tortonian (Bouchet 2009). The assemblage no. 4 is composed of rare late Cretaceous fossils (Squalicorax sp., Cretolamna sp., Ptychodus sp.) reworked in the Miocene shelly sand. These fossils are too damaged for providing more detailed information. The assemblage no. 5 contains a vertebrate fauna from the early Orleanian (MN3): Ptychogaster sp., Testudo sp., Trionyx sp., Diplocynodon sp., Lagopsis sp., Eucricetodon infralactorensis, Steneofiber depereti, Amphicyon (Megamphicyon) lathanicus, Cynelos helbingi, Cynelos schlosseri, Haplocyonoides mordax, Ballusia hareni, Phoberocyon aurelianensis, Phoberocyon dehmi, Plithocyon bruneti, Hemicyon gargan, Martes laevidens, Palaeogale hyaenoides, Plesiogale angustifrons, Pseudaelurus turnauensis, Anchitherium aurelianense, Protaceratherium minutum, Diaceratherium aurelianense (Fig. 6), Aureliachoerus aurelianensis, Xenohyus venitor, Brachyodus onoideus, Brachyodus intermedius, Procervulus dichotomus, Heterocemas sp., Ligeromeryx praestans and Andegameryx andegaviensis. These fossils have undergone a very low reworking as proven by their good state of preservation and the presence of a fluvial sand lens from the early Orleanian in the eastern part of the paleontological site (Fig. 5). A larger set of late Orleanian (MN5) fossils composes the assemblage no. 6: Chelydropsis sp., Ptychogaster sp., Testudo promarginata, Testudo sp., Trionyx sp., Lacertidae indet., Boidae indet., Colubridae indet., Viperidae indet., Diplocynodon styriacus, Diplocynodon sp., Tomistoma cf. lusitanica, Gavialis sp., Incertae sedis, Proscapanus sansaniensis, Lagopsis sp., Steneofiber depereti, Anchitheriomys wiedemanni, Pliopithecus piveteaui, Hyainailouros sulzeri, Amphicyon giganteus, Agnotherium grivensis, Pseudocyon sansaniensis, Pseudarctos bavaricus, Hemicyon sansaniensis, Trocharion albanense, Protictitherium gaillardia, Pseudaelurus romievensis, Pseudaelurus lorteti, Pseudaelurus turnauensis, Prodeinotherium bavaricum, Gomphotherium angustidens, Zygolophodon turicensis, Anchitherium aurelianense, Anisodon grande, Brachypotherium brachypus, Plesiaceratherium lumiarense, Prosantorhinus germanicus, Prosantorhinus douvillei, Lartetotherium sansaniensis, Aureliachoerus aurelianensis, Bunolistriodon lockharti, Hyotherium soemmeringi, Dorcatherium guntianum, Dorcatherium crassum, Amphimoschus pontileviensis, Dicrocerus elegans, Lagomeryx sp. and Palaeomeryx bojani. These fossils are all reworked from an unknown continental facies. Despite the poor state of conservation of the fossils, this assemblage no. 6 is consistent (Fig. 8). All these taxa appear in the fossil land mammals list defined by Ginsburg (2001) for the Miocene deposits (MN5) of the Savigné-sur-Lathan/Noyantsous-le-Lude Basin (France).

\section{INTRODUCTION}

En Anjou-Touraine, de nombreux gisements paléontologiques à vertébrés cénozoïques ont déja été publiés dont les principaux sont: Les Beilleaux (Ginsburg 1989b), Pontigné (Ginsburg \& Bonneau 1995), La Roche-Ragot (Ginsburg et al. 1978), La Guimardière (Gagnaison et al. 2004), Pelmer (Bouchet et al. 2010), La Brosse et Mauvières (Ginsburg et al. 2000). Dans ces travaux, les études paléontologiques (taxonomie et biostratigraphie) sont très détaillées. Par contre, les descriptions des contextes géologiques, stratigraphiques et taphonomiques sont souvent présentées en second plan lors de la description de ces sites paléontologiques.

Le site paléontologique du Grand Morier est situé en plein cœur du golfe ligérien à 40 kilomètres à l'ouest de la ville de Tours (Temey 1996). Ce gîte fossilifère correspond à une ancienne carrière d'exploitation de falun miocène (Fig. 1). Les coordonnées GPS du centre de cette falunière sont: $47^{\circ} 19^{\prime} 32.74{ }^{\prime \prime} \mathrm{N}, 0^{\circ} 19^{\prime} 40.92$ ' E; l'altitude du site varie entre 64 et 76 mètres. Cette petite relique géologique de sédiments miocènes est complétement isolée et déconnectée du bassin de Savigné-sur-Lathan/Noyant-sous-le-Lude situé à 10 kilomètres plus au nord (Alcaydé 1975; Andrin \& Mathivon 2015). Plus précisément, cette ancienne falunière se situe à six kilomètres à l'ouest de la ville de Langeais, à quatre kilomètres au nord de la commune de Saint-Michel-sur-Loire et à un kilomètre au sud du hameau de Pont-Boutard (Fig. 2). L'exploitation de la carrière s'est surtout développée pendant tout le XXème siècle pour l'utilisation du falun dans la construction de voirie ou pour le chaulage (Lecointre 1947). Sa surface totale d'exploitation avoisine les 6 hectares. Actuellement, le site a été réhabilité sous la forme d'un grand champ pour l'agriculture. Des restes de fronts de taille sont encore visibles dans la partie boisée longeant la route départementale D125 (Fig. 2). Dès l'ouverture de la falunière, de nombreux paléontologues (professionnels et amateurs) sont venus prélever des fossiles de vertébrés.

Les travaux géologiques et paléontologiques les plus notables réalisés à propos du site du Grand Morier sont ceux de Lecointre (1930, 1947), Alcaydé (1975) et Alcaydé et al. 
(1976) pour les levers géologiques (cartographie, stratigraphie et tectonique), et ceux de Buge (1948) et Ginsburg (2001) pour leurs travaux sur les fossiles (respectivement les bryozoaires et les mammifères terrestres).

Cet article présente des données inédites au niveau du contexte géologique et de la stratigraphie des séries sédimentaires cénozoïques du site du Grand Morier. Depuis 1990 jusqu’à nos jours, des observations et des levers géologiques ont été faits dans la falunière mais aussi tout autour du hameau de Pont Boutard (Andrin \& Mathivon 2015) afin de réviser la carte géologique dessinée par Alcaydé (1975). Ces levers de terrain permettent de mieux comprendre l'agencement des différents corps sédimentaires par rapport à la tectonique locale. En parallèle, de nombreuses collections paléontologiques ont été visitées pour compléter la liste taxonomique de vertébrés fossiles proposée par Ginsburg (2001) et pour proposer un découpage stratigraphique détaillé des différentes formations cénozoïques visibles sur le site du Grand Morier. Pour cela, des assemblages de vertébrés fossiles ont été reconstitués pour chaque couche géologique en suivant la méthodologie déjà utilisée pour les sites paléontologiques de Pelmer et de La Guimardière (Maine-et-Loire, Gagnaison 2013).

\section{MATÉRIEL ET MÉTHODES}

Cette étude se base sur des observations faites sur des fossiles provenant de multiples collections visitées par l'auteur depuis 1992 jusqu'à nos jours. La majorité provient de collections paléontologiques de différents musées: MNHN de Paris, MHN de Tours, Musée du Savignéen à Savignésur-Lathan (collections Quétin, Le Déoré et Marchand). Quelques fossiles proviennent de nombreuses collections privées: P.-A. Gillet, M. Dechamps, J.-C. \& C. Gagnaison, B. Cossard, B. Guevel, J.-J. Leroux, D. Sossong, S. Xerri, E. Métais, F. Quatrehomme, J.-M. Sinturet, F. Cahoreau, F. Chevrier, G. Barbe. Aucun inventaire détaillé de l'ensemble des fossiles de vertébrés étudiés n'a été réalisé. Seule la liste faunistique présentée dans ce travail a été faite (Annexe 3), mais il est possible d'estimer le nombre de spécimens étudiés pour le site paléontologique du Grand Morier à environ 400 dents et 50 os. Cette série d'observations complète la liste faunique proposée par Ginsburg (2001) pour ce gisement. Cette dernière est composée de 53 taxons de mammifères terrestres. Toutes les déterminations des fossiles des collections privées ont été faites et/ou vérifiées par L. Ginsburg (MNHN, Paris) et/ou par C. \& J.-C. Gagnaison (respectivement Institut Polytechnique UniLaSalle Beauvais \& Musée du Savignéen) et F. Chevrier (MHN Orléans). De nombreuses comparaisons ont été effectuées avec les fossiles publiés dans les nombreuses publications sur ce sujet (voir la liste des références bibliographiques); ainsi qu'avec les fossiles conservés dans les riches collections paléontologiques du Muséum national d'Histoire naturelle de Paris (MNHN), du Musée du Savignéen (Savigné-sur-Lathan, Indre-et-Loire, musée de France depuis 2003) et des collections historiques de l'Institut Polytechnique UniLaSalle (Beauvais, Oise).
La majorité (90\%) des fossiles étudiés s'accompagne d'informations sur leur contexte géologique: soit les observations ont été faites directement sur le terrain pendant les nombreuses visites effectuées au Grand Morier entre les années 1990 et 1997 ; soit les informations ont été notées directement sur les étiquettes accompagnant les fossiles dans les différentes collections. Une grande partie du matériel étudié a été nettoyée et observée sous une loupe binoculaire afin de trouver des traces d'altération et des résidus de sédiments. Toutes ces informations - géologiques, taphonomiques et taxonomiques - ont été prises en compte afin de créer des assemblages de vertébrés crétacés et cénozoïques. Seuls les fossiles dont toutes les données étaient fiables ont été utilisés pour la définition de ces assemblages.

\begin{tabular}{|c|c|}
\hline \multicolumn{2}{|c|}{ ABRÉVIATIONS } \\
\hline 994 & $\begin{array}{l}\text { Fossile enregistré en } 1994 \text { dans les collections du Muséc } \\
\text { du Savignéen (Savigné-sur-Lathan, Indre-et-Loire); }\end{array}$ \\
\hline 2012 & $\begin{array}{l}\text { fossile enregistré en } 2012 \text { dans les collections du Musé } \\
\text { du Savignéen (Savigné-sur-Lathan, Indre-et-Loire); }\end{array}$ \\
\hline $\mathrm{C} / \mathrm{c}$ & canine; \\
\hline $\mathrm{D} / \mathrm{d}$ & dent déciduale; \\
\hline d. & droite; \\
\hline g. & gauche; \\
\hline $\mathrm{I} / \mathrm{i}$ & incisive; \\
\hline LB & $\begin{array}{l}\text { fossile enregistré dans les collections paléontologique de } \\
\text { l'Institut polytechnique UniLaSalle (Beauvais, Oise) }\end{array}$ \\
\hline $\mathrm{M} / \mathrm{m}$ & molaire supérieure/inférieure; \\
\hline MHN & Muséum d'Histoire naturelle, Tours; \\
\hline MNHN & Muséum national d'Histoire naturelle; \\
\hline $\mathrm{P} / \mathrm{p}$ & prémolaire supérieure/inférieure; \\
\hline PBT & Le Grand Morier (Pont-Boutard, Indre-et-Loire). \\
\hline
\end{tabular}

\section{CONTEXTE GÉOLOGIQUE}

Aucun affleurement naturel n'est visible autour du hameau de Pont-Boutard. Les observations géologiques de terrain se font sur des strates en place: sur les fronts de taille des carrières ou à la faveur de travaux de construction de bâtiments et de voirie. Des compléments d'informations ont été faits grâce à des échantillons isolés lors de prélèvements faits à l'aide d'une tarière ou directement sur les pierres volantes visibles dans les champs (Andrin \& Mathivon 2015). La première étude géologique détaillée est proposée lors du lever de la carte géologique de Tours au 1/80 000 (Lecointre 1930). Ces premières observations permettent de mettre en évidence à la fois la série stratigraphique du Crétacé au Pliocène et la présence d'une importante faille verticale de direction nord-sud dans cette région qui sera nommée «la faille de Pont-Boutard" (Lecointre 1947; Alcaydé et al. 1976). Lors du lever de la carte géologique de Chinon au 1/50000, Alcaydé (1975) va retravailler la géologie de Pont-Boutard en réalisant deux principaux changements par rapport aux travaux de Lecointre $(1930,1947)$ : la «faille de Pont-Boutard » est dessinée avec une direction nord-est/ sud-ouest et un important placage de calcaire lacustre du "Ludien inférieur» (Annexe 1) est placé au milieu des deux témoins de faluns miocènes. Après cette date et jusqu'à la fin du XXème siècle, plusieurs carrières se sont ouvertes et/ 


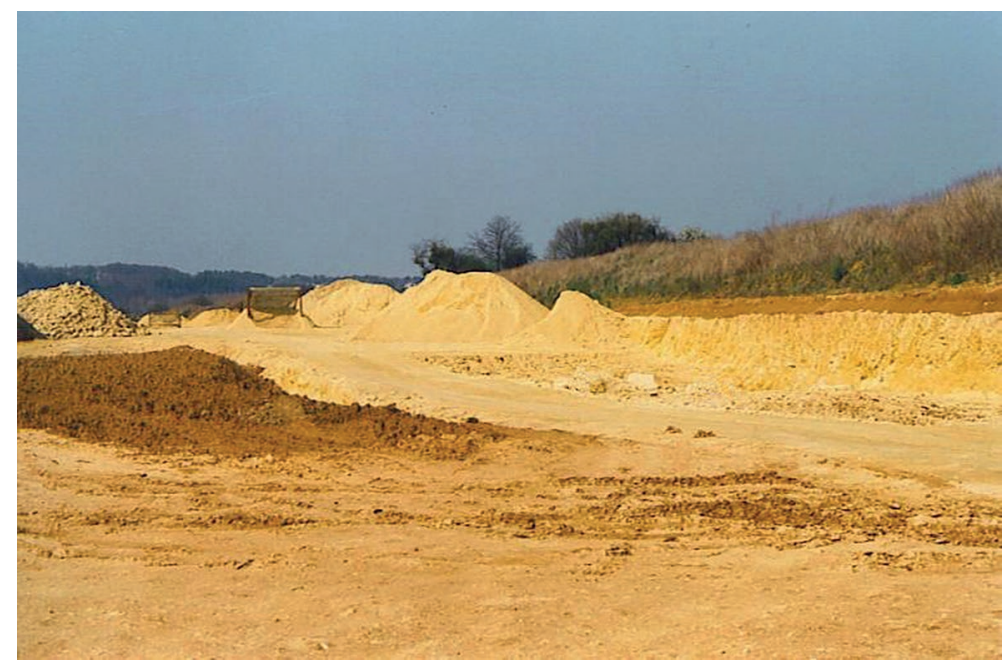

FIG. 1. - Vue générale de la falunière du Grand Morier (Pont-Boutard, Indre-et-Loire, France) en exploitation en 1994.

ou étendues autour du hameau de Pont-Boutard dont: la falunière du Grand Morier (les faluns du Miocène), les carrières de kaolin du Petit Morier et de La Rouchouze (les argiles du Sénonien) et la falunière de Pont-Boutard (les faluns de Continvoir du Turonien supérieur). En parallèle, des travaux de construction de maisons individuelles ont permis de voir ponctuellement les formations crétacées au niveau des hameaux de Pont-Boutard et de La Rouchouze (le tuffeau du Turonien supérieur et les argiles du Sénonien). Pendant les travaux de construction de l'autoroute A85, il a été possible de faire des observations géologiques sur les sables à graviers (Miocène terminal-Pliocène) ainsi que sur les formations superficielles (Quaternaire-Actuel). Toutes ces dernières observations contredisent les levers géologiques de Lecointre (1930, 1947), d'Alcaydé (1975) et d'Alcaydé et al. (1976). Une nouvelle campagne géologique a été lancée pendant le mois de septembre 2015 par l'Institut Polytechnique UniLaSalle Beauvais afin de revoir entièrement le contexte géologique autour du hameau de PontBoutard. Un ensemble de 168 observations géologiques ont été faites sur les anciens fronts de taille des carrières et sur des prélèvements faits à l'aide d'une tarière manuelle (Andrin \& Mathivon 2015). La synthèse de ces données permet de proposer une carte géologique inédite, corrigée et beaucoup plus précise que les précédentes (Fig. 2). Ce travail permet de corriger le dessin des enveloppes des corps sédimentaires et de préciser l'allure des failles présentes autour du hameau de Pont-Boutard. Les deux principales améliorations au niveau du dessin des corps sédimentaires sont: la suppression des calcaires lacustres ludiens au sud de Pont-Boutard et le rajout des formations superficielles (alluvions et colluvions). Au niveau du contexte tectonique, les observations de terrain couplées avec les observations des photographies aériennes ont montré à la fois que la «faille de Pont-Boutard " avait une direction azimutale Nord10 et que cet accident était accompagné par d'autres failles (de direction azimutale comprise entre Nord150 et Nord40) décalant les séries sédimentaires du Crétacé à l'Éocène incluses et cachetées par les formations du Miocène-Actuel. En synthèse, ces failles - verticales à composante normale - forment une structure en graben dont le cœur est rempli par les séries sédimentaires du Cénozoïque (Fig. 3).

\section{LES DIFFÉRENTES FORMATIONS SÉDIMENTAIRES}

Tout autour de Pont-Boutard, l'assise crétacée (TuronienSénonien) est recouverte par huit formations sédimentaires du Cénozoïque ainsi que par des formations superficielles récentes (Fig. 4).

\section{LE SUBSTRAT CRÉTACÉ}

Trois faciès marins du Crétacé supérieur sont visibles autour du village de Pont-Boutard (Alcaydé \& Rasplus 1971; Alcaydé 1975). L'ensemble de ces formations crétacées représente un substrat calcaire affleurant sur une puissance avoisinant la vingtaine de mètres. Le Turonien supérieur est représenté par un tuffeau bioclastique jaune et massif au nord de Pont-Boutard (Alcaydé 1975). Ce faciès massif passe latéralement à une formation nommée le «falun de Continvoir» (Desforges \& Merlu 2010). Ce sédiment meuble est bien visible dans l'ancienne carrière au sud-ouest de Pont-Boutard. Le Turonien supérieur est toujours recouvert par une formation argileuse du Sénonien. Cette argile kaolinique est riche en silex, en spongolites et en fossiles silicifiés (spongiaires, oursins, brachiopodes, mollusques; Dourlat \& Emeriau 2012). La surface de contact entre les formations du Crétacé et celles du Cénozoïque est souvent irrégulière et érosive. Toutes les formations du Cénozoïque sont discordantes sur le Crétacé. La plus forte discordance angulaire est marquée par les formations néogènes sur les argiles à spongiaires du Sénonien au niveau des hameaux du Petit Morier et du Grand Morier (Fig. 2). 
LE CONGLOMÉRAT SILICEUX (CRÉTACÉ TERMINAL-ÉOCÈNE) Anciennement, ce conglomérat portait le nom de "perrons de Touraine» (Hom \& Moulin 2012). En général, cette formation chapeaute les sommets des collines constituées de sédiments crétacés (Fig. 2). Elle a aussi été observée sous la forme de gros blocs dans la partie nord de l'ancienne falunière du Grand Morier. Plusieurs de ces gros blocs sont encore visibles au début de l'entrée nord de cette ancienne carrière. La puissance de cette formation varie entre 0,5 et 8,5 mètres. Stratigraphiquement, elle est comprise entre les derniers dépôts du Crétacé (les argiles à spongiaires du Sénonien) et les premiers dépôts du Paléogène (l'argile verte de l'Éocène). Aucun critère stratigraphique précis n'a encore été trouvé dans cette formation. Sa couleur varie entre le marron foncé et le rouge vif (Alcaydé 1975). Ce conglomérat est plus ou moins bien cimenté. Il peut se présenter sous la forme de petits morceaux noyés dans une argile marron ou encore sous la forme de grandes dalles silicifiées de plusieurs mètres-carrés. Les éléments constituant ce conglomérat sont principalement des silex, des spongolites, des débris d'éponges siliceuses remaniées du Crétacé et de rares dragées de quartz laiteux. Le ciment est siliceux (calcédoine et opale) ou argileux. Lors des analyses microfaciologiques, de très nombreux éléments pédogénétiques ont été observés: traces de racines, manchons racinaires et pisolithes vadoses (Hom \& Moulin 2012). La genèse de ce conglomérat est donc liée à une mise en place d'un paléosol (Alcaydé 1975) depuis la fin du Crétacé jusqu'à l'Éocène comme dans de très nombreuses régions d'Europe occidentale.

\section{L'ARGILE SABLEUSE VERTE (ÉOCÈNE)}

Autour du hameau de Pont-Boutard, cette formation sédimentaire n'est visible qu'au niveau de l'ancienne falunière du Grand Morier. Pendant l'exploitation du falun miocène, les carriers atteignaient régulièrement l'argile sableuse verte située directement sous le conglomérat miocène ou sous le calcaire lacustre (Fig. 5). Cette formation est encore visible sur une puissance de quelques décimètres au niveau de l'ancien front de taille adjacent à l'entrée nord de l'ancienne falunière. Sur cet affleurement, cette formation éocène est en contact direct avec le conglomérat miocène. La surface de contact avec le conglomérat siliceux sous-jacent n'a jamais été observée. La puissance maximale vue à l'affleurement est de 2,5 mètres dans la partie est de l'ancienne falunière. Cette argile est plastique et de couleur vert pâle. Elle contient de nombreux débris siliceux de la taille des silts et des sables fins: des grains de quartz et des spicules de spongiaires remaniés des formations crétacées. Aucune structure sédimentaire n’a été observée. Un seul fossile a été trouvé: une dent de Plagiolophus cf. annectens (Owen, 1847) (Gagnaison 2007). En Anjou-Touraine, cette formation continentale est considérée comme un dépôt fluvio-lacustre à fluviatile de faible énergie (Ginsburg et al. 1978; Hom \& Moulin 2012). Ce faciès est identique à celui du gisement paléontologique de La Roche-Ragot à Milly-le-Meugon (Anjou) où la riche faune de vertébrés terrestres a permis de donner un âge éocène supérieur ( Ludien ", Annexe 1) à cette formation (Ginsburg et al. 1978).
LE CALCAIRE LACUSTRE DE TOURAINE

(OLIGOCÈNE-AGÉNIEN SUPÉRIEUR)

Ce faciès n'a été observé qu'au niveau du chemin nord pendant l'exploitation de la falunière du Grand Morier (Fig. 5). Sa puissance n'excède pas le mètre. Ce calcaire est toujours compris entre l'argile verte de l'Éocène et le conglomérat miocène (Fig. 5). La surface de contact avec l'Éocène est nette. Ce calcaire est blanc, massif et bioclastique (Alcaydé \& Rasplus 1971). Il contient quelques coquilles indéterminables d'ostracodes et de mollusques pulmonés lacustres (planorbes et limnées). Le toit de cette formation est irrégulier. Il est marqué par de nombreux trous centimétriques à pluridécimétriques coniques liés à une décalcification du calcaire par une altération atmosphérique. Ces perforations sont remplies d'une argile plastique noire et compacte pouvant atteindre une épaisseur maximale de 50 centimètres. Dans cette argile, les paléontologues amateurs trouvaient régulièrement des fragments d'os et des dents noires de mammifères appartenant principalement à Protaceratherium minutum (Cuvier 18221824). Cette surface est aussi recouverte d'animaux marins encroûtants (bryozoaires, huitres, coraux, balanes) et de trous creusés par des bivalves lithophages marquant la mise en place du conglomérat marin du Miocène. Historiquement, ce calcaire nommé "calcaire lacustre de Touraine» est daté de l'Oligocène («Stampien inférieur»; Alcaydé \& Rasplus 1971; Yvard 1976, Annexe 1), mais la présence de la petite forme de $P$. minutum dans les poches de décalcification prolonge l'âge de ce calcaire jusque dans l'Agénien supérieur (Ménouret \& Guérin 2009; Antoine \& Becker 2013). Cet âge est compatible avec les calcaires lacustres du Blésois (Ginsburg \& Hugueney 1980 ; Scherler et al. 2013).

\section{LE SABLE FLUVIATILE (ORLÉANIEN INFÉRIEUR)}

Cette formation sédimentaire n'a été visible qu'une seule fois en 1995 dans la partie sud de la carrière de faluns du Grand Morier (Fig. 5). Une lentille de ce sable continental était conservée entre les argiles vertes de l'Éocène et le conglomérat miocène. Cette poche s'étendait sur une largeur avoisinant 1,2 mètre et sur une hauteur d'environ 0,80 mètre. Sa forme générale pouvait faire penser à une structure chenalisée. Aucune strate oblique n'a été observée. Le sable est grossier et de couleur grise. Il est composé de $95 \%$ de grains de quartz laiteux à gris, de $5 \%$ d'argiles et de quelques traces d'oxydes indéterminables. Ce sable est identique à celui observé dans le site de La Guimardière (Gagnaison 2013). Aucun fossile n'a été trouvé dans cette formation. En Anjou-Touraine, ce faciès continental correspond à un dépôt sédimentaire fluviatile daté de l'Orléanien inférieur (Gagnaison 2013; Cabidoche \& Riera 2013). Un échantillon de ce sable fluviatile est conservé dans les collections géologiques du Musée du Savignéen à Savigné-sur-Lathan (Indre-et-Loire).

\section{LA CALCARÉNITE À STRATES OBLIQUES}

\section{(BURDIGALIEN SUPÉRIEUR-LANGHIEN INFÉRIEUR)}

Cette calcarénite n'a été observée que dans la partie nordest de l'ancienne falunière du Grand Morier (Fig. 5) entre les années 1990 et 1995. Sa puissance maximale n'excédait 


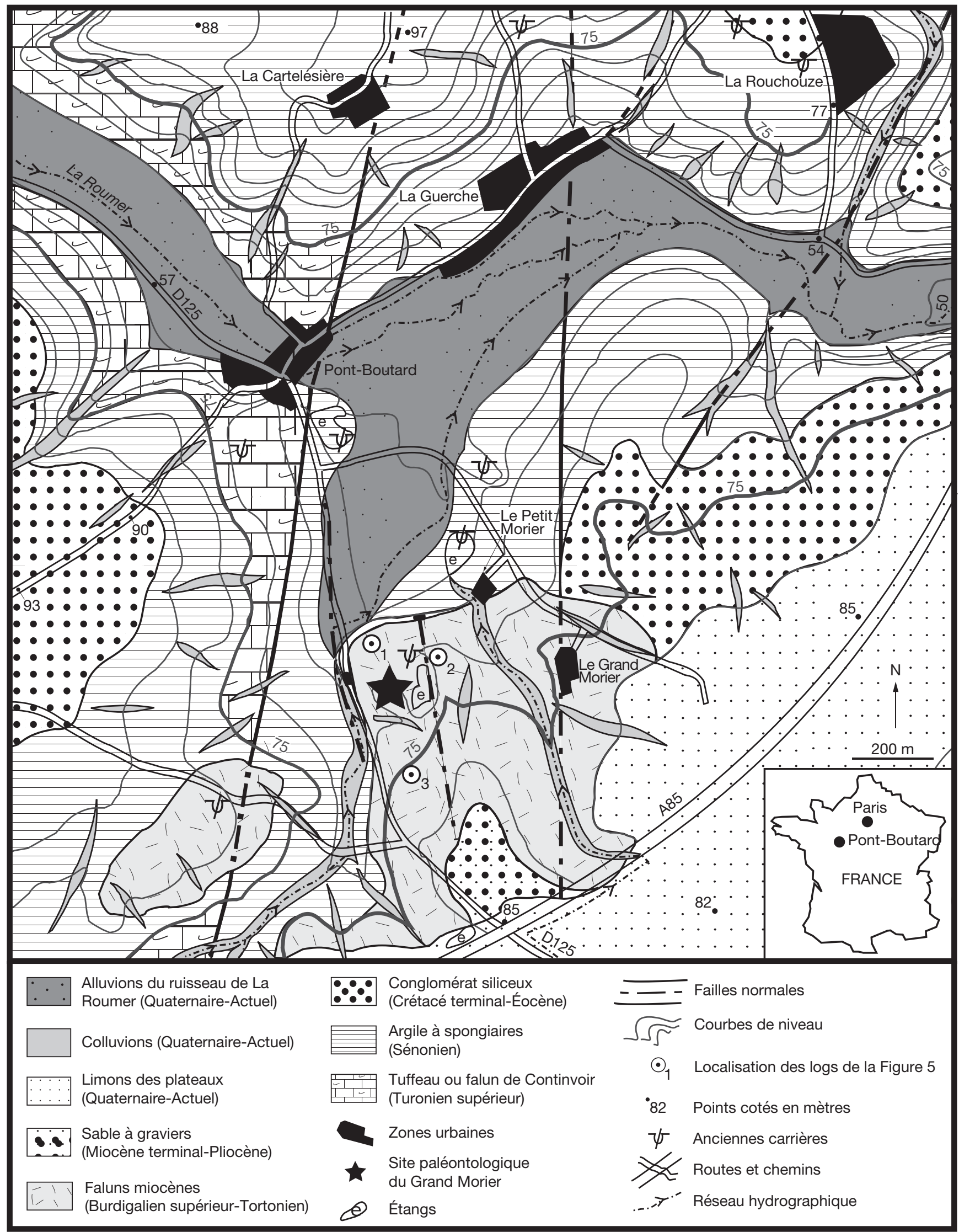


pas 1,30 mètre. Cette formation était comprise entre l'argile sableuse verte éocène et le conglomérat de base du falun à bryozoaires. Elle se biseaute vers le sud au bout d'une quinzaine de mètres. Son mur est érosif et présente quelques petits graviers de silex ainsi que des galets mous provenant du remaniement de la formation éocène. La calcarénite est de couleur jaune et se débite en plaquettes décimétriques. Elle est principalement bioclastique (mollusques, bryozoaires, balanes, dents de téléostéens et de chondrichthyens). Les grains de quartz sont rares et fortement roulés. Les rares fossiles complets sont des oursins plats du genre Scutella. De nombreuses strates obliques entrecroisées sont visibles et indiquent un courant bidirectionnel suivant la direction nord-sud. Cette calcarénite à oursins plats est connue dans le bassin sédimentaire de Savigné-sur-Lathan/Noyant-sous-le-Lude (Hom \& Moulin 2012). Elle correspond à la première transgression marine du Miocène datée du Burdigalien supérieur-Langhien inférieur (Courville \& Bongrain 2003).

\section{LE CONGLOMÉRAT DE BASE DU FALUN À BRYOZOAIRES (LANGHIEN-TORTONIEN INFÉRIEUR)}

Cette formation miocène a été observée dans toute l'ancienne falunière du Grand Morier ainsi que sur le petit témoin miocène de La Boutarderie (Fig. 1). Actuellement, il est toujours visible dans la partie boisée du site du Grand Morier à proximité du chemin d'accès à l'étang. Sa puissance varie entre 0,2 et 1,5 mètre. Sa base est érosive sur toutes les formations géologiques antérieures. Aucune structure sédimentaire et tectonique n'a été observée dans cette formation. Dans la partie orientale de l'ancienne falunière du Grand Morier, ce conglomérat est fortement cimenté par de la sparite (Fig. 6). Ce conglomérat contient des éléments de différentes natures : des galets de calcaires lacustres perforés par des mollusques lithophages et recouverts de bryozoaires, des galets du conglomérat siliceux du Crétacé-Éocène, des éléments siliceux roulés (silex et spongiaires remaniés du Sénonien), de grands morceaux de bivalves miocènes (ostréidés, pectinidés) et des restes de vertébrés marins (téléostéens, chondrichthyens et mammifères) et continentaux (os et dents de mammifères et reptiles). Le diamètre de ces objets varie entre quelques centimètres et 0,8 mètre pour les plus gros. Cette formation peut se rapprocher des faluns à graviers du bassin sédimentaire de Savigné-sur-Lathan/Noyant-sous-le-Lude daté du LanghienTortonien inférieur (Courville \& Bongrain 2003; Gagnaison 2013). Toutes ces observations marquent un environnement marin de faible profondeur (intertidal à infralittoral supérieur) qui subissait des courants de fond de forte énergie.

\section{LE FALUN À BRYOZOAIRES (TORTONIEN)}

Il s'agit du faciès qui était exploité dans l'ancienne falunière pour les travaux de voirie et le chaulage. Sa puissance varie entre 1,0 et 6,0 mètres. Sa puissance maximale était visible à l'emplacement de l'étang actuel au début des années 1990. Ce falun affleure toujours sur une puissance de 3,5 mètres dans la partie occidentale boisée de l'ancienne falunière (Fig. 5). Ce falun jaune présente localement une cimentation calcitique (sparite hétérogranulaire) donnant cet aspect massif à l'affleurement. Il est composé à plus de $95 \%$ de bioclastes marins de toutes natures (bryozoaires, mollusques, échinodermes, dents de téléostéens et de chondrichthyens) et de quelques rares lithoclastes (des grains de quartz, des galets de silex et des petits galets de calcaire perforés). Buge (1948) décrit de grandes strates obliques progradantes vers le sud à la base de cette formation. Entre 1980 et 1995, seuls des granoclassements positifs de faible ampleur et des lamines horizontales grossières ont été observées. Ce faciès marin correspondait à une ancienne prairie sous-marine à bryozoaires de l'infralittoral supérieur (Gagnaison 2012) du Tortonien (Courville \& Bongrain 2003).

\section{LE SABLE À GRAVIERS (MiocÈNE TERMINAL-PLIOCÈNE)}

Cette formation se voit uniquement à l'intersection entre l'autoroute A85 et la départementale D125 en direction de Pont-Boutard (Fig. 2). Lors des travaux de cet aménagement routier, ce sable à graviers était visible sur une puissance de 4,5 mètres. Actuellement, il est toujours possible de trouver de nombreux graviers dans les labours des champs avoisinants l'autoroute A85. Cette formation est directement posée sur la partie supérieure des faluns à bryozoaires et recouverte par les limons des plateaux. C'est un sable grossier argileux riche en grains de quartz et en graviers de toutes natures (quartz, silex, fossiles crétacés remaniés et roulés). Sa couleur varie entre le jaune pâle et l'orange. Un fragment de bois fossile roulé et silicifié indéterminable a été trouvé dans cette formation. Ce sable correspond à un épandage détritique fluviatile de la fin du Néogène (Miocène terminal-Pliocène) (Alcaydé \& Rasplus 1971).

\section{LES FORMATIONS SUPERFICIELLES : LES LIMONS DES PLATEAUX, LES COLLUVIONS ET LES ALLUVIONS (QUATERNAIRE-ACTUEL)}

La formation éolienne des limons des plateaux est épaisse de quelques mètres (Fig. 2). Elle se développe sur tout le plateau surplombant la Loire entre les villes de Saint Patrice, de Langeais et de la commune de Saint Michel-sur-Loire. Ces limons recouvrent en discordance toutes les formations du Crétacé au Néogène (Alcaydé 1975). Les colluvions correspondent à des loupes de glissement plurimétriques - visibles sur tous les versants - mélangeant de nombreux blocs de différentes natures (perrons, tuffeau, calcaire lacustre, calcarénite, silex) dans des argiles plastiques de couleur marron. La plaine alluviale du ruisseau de La Roumer forme une plaine marécageuse autour du hameau de Pont-Boutard. Cette dernière formation superficielle est un mélange de sables, d'argiles et de galets polygéniques dont l'épaisseur ne dépasse pas quelques mètres (Alcaydé 1975).

\section{L'INFLUENCE DE LA TECTONIQUE SUR LA MISE EN PLACE DES DÉPÔTS SÉDIMENTAIRES DU CÉNOZOÏQUE}

La tectonique de l'ouest de la Touraine est marqué par la présence du graben de Langeais affectant principalement les séries crétacées (Desforges \& Merlu 2010). Les grandes failles - de direction nord-sud et à composante normale bordant cette grande structure tectonique - sont des accidents hercyn- 


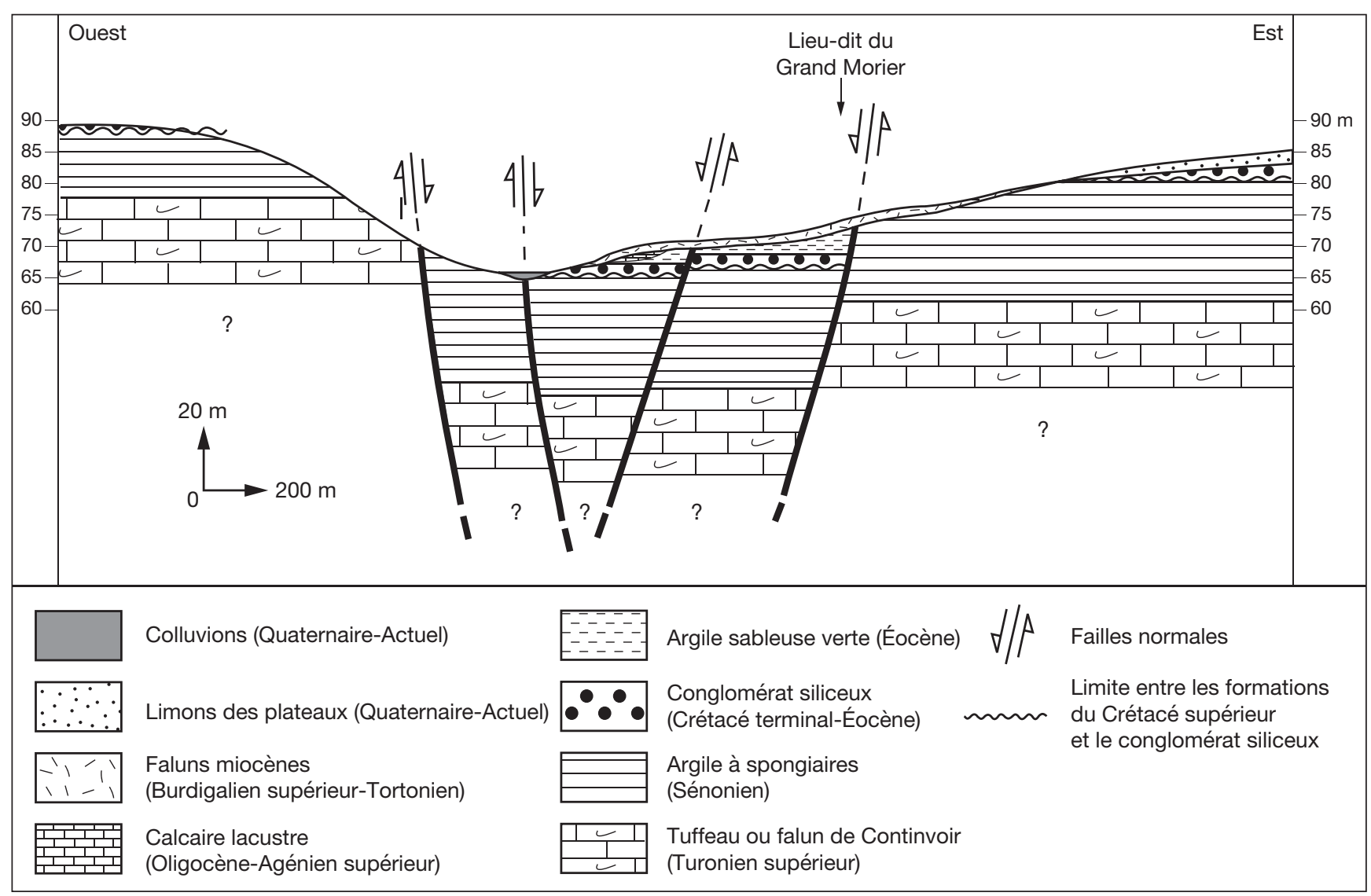

FIG. 3. - Coupe géologique de direction ouest-est extraite de la carte géologique de Pont-Boutard (Fig. 2) passant par le site paléontologique du Grand Morier. L'échelle des altitudes est exagérée volontairement pour améliorer la lisibilité du graben de Pont-Boutard.

iens réactivés pendant les différentes phases de la tectonique pyrénéo-alpine (Debelmas 1974). La faille bordant l'ouest du graben de Langeais correspond à la "faille de Pont-Boutard" (Alcaydé et al. 1976). Le graben de Pont-Boutard est une structure tectonique secondaire s'accrochant sur la partie ouest du bloc effondré du graben de Langeais. L'histoire tectonique du graben de Pont-Boutard peut se diviser en deux principaux épisodes: la tectonique fin Crétacé-Paléogène (phase pyrénéenne) et la tectonique syn-transgression miocène (phase alpine). À la fin du Crétacé, toute la région de Pont-Boutard est émergée permettant la mise en place d'un milieu continental recouvert d'un sol dont l'épaisseur est globalement homogène (Alcaydé 1975). Cette pédogenèse se poursuit jusqu'au début de l'Éocène (Alcaydé \& Rasplus 1971). La phase pyrénéenne correspond localement à l'activation de la «faille de Pont-Boutard " et de celles passant par le lieu-dit du Grand Morier et à proximité du hameau de La Rouchouze au cours de l'Éocène; formant ainsi le graben de Pont-Boutard selon une direction globalement nord-sud (Fig. 3). Entre l'Éocène et l'Orléanien inférieur, trois formations continentales vont se développer exclusivement dans cette cuvette morphologique: un réseau fluviatile pendant l'Éocène, un lac pendant l'Oligocène-Agénien supérieur et un second réseau fluviatile pendant l'Orléanien inférieur (Alcaydé 1975). Pendant le Burdigalien (phase alpine), deux petites failles normales secondaires de petite ampleur vont se développer au cœur du graben de Pont-Boutard permettant son approfondissement. Ces petites failles vont casser et décaler les formations tertiaires précédentes (Fig. 3). La première transgression marine (Burdigalien supérieur-Langhien inférieur) va transgresser depuis le bassin de Savigné-sur-Lathan/Noyant-sous-le-Lude vers la Touraine du Sud grâce à ce couloir naturel. La seconde transgression miocène (Langhien à Tortonien) va être - elleaussi - canalisée par ce couloir morphologique sous-marin. Par contre, cette seconde transgression va combler et recouvrir entièrement, en on-lap, toute cette structure tectonique sans être affectée par les failles (Fig. 3). Les derniers dépôts sédimentaires entre le Miocène terminal et l'actuel, continuent ce recouvrement sans être affectés par la tectonique.

\section{TAPHONOMIE}

Les observations faites sur l'ensemble des fossiles font ressortir quatre contextes taphonomiques.

\section{LES FOSSILE DES ARGILES VERTES (ÉOCÈNE)}

Le seul fossile trouvé dans ce faciès correspond à la dent de $P$. cf. annectens (Fig. 7). Lors de sa découverte, ce fossile était posé sur un tas composé d'argile sableuse éocène et de 


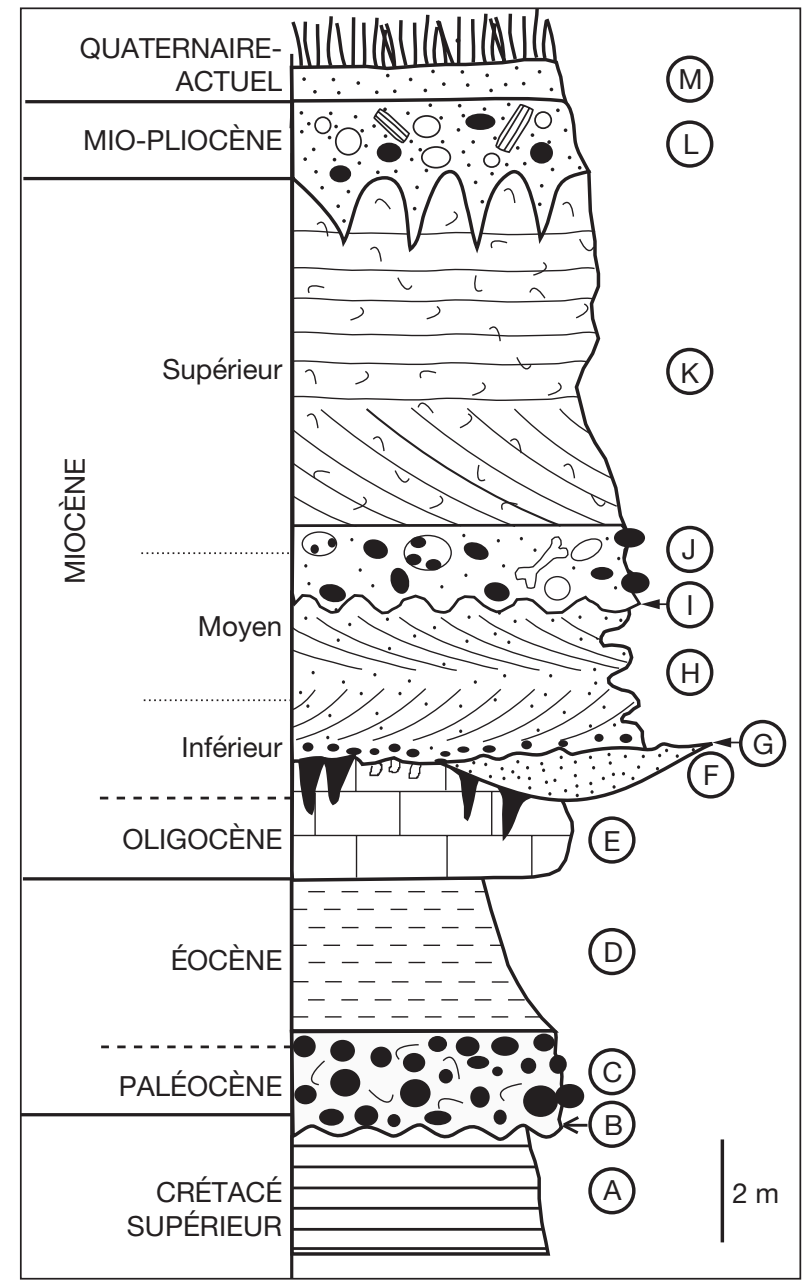

FIG. 4. - Colonne lithostratigraphique synthétique des différentes formations sédimentaires du site paléontologique du Grand Morier: A, argile à spongiaires (Sénonien); B, surface de contact entre les formations du Crétacé supérieur et le conglomérat siliceux; $\mathbf{C}$, conglomérat siliceux (Crétacé terminal-Éocène); D, argile sableuse verte (Éocène); E, calcaire lacustre avec ses poches d'argile noire de décalcification à son sommet (Oligocène-Agénien supérieur); $\mathbf{F}$, sable continental (Orléanien inférieur); $\mathbf{G}$, surface érosive de la transgression miocène; $\mathbf{H}$, calcarénite à strates oblique (Burdigalien supérieurLanghien inférieur); I, surface érosive entre la calcarénite à strates obliques et le conglomérat miocène; $\mathbf{J}$, conglomérat de la base des faluns à bryozoaires (Langhien-Tortonien inférieur); $\mathbf{K}$, falun à bryozoaires (Tortonien); L, sable à graviers (Miocène terminal-Pliocène); $\mathbf{M}$, limons des plateaux chapeautés par la terre végétale actuelle (Quaternaire-Actuel).

faluns miocène. Dans un premier temps, elle fut considérée comme remaniée dans les faluns miocènes (Gagnaison 2007). Après un nouvel examen du fossile, des restes d'argile verte sont encore visibles entre ses racines. De plus, lors de sa découverte, sa couronne dentaire était recouverte d'une gangue argileuse verte. Ses racines présentent des cassures récentes liées aux engins d'extraction. La couronne est de couleur jaune et les racines sont marrons. Cette dent est bien conservée et n'est ni patinée ni roulée. De nombreuses petites fissures sont visibles au niveau de l'émail. Aucun indice d'altération liée à un transport important n'est visible. Cette dent a du se déposer dans un milieu lacustre calme au cours de l'Éocène.
LES FOSSILES DE VERTÉBRÉS CONTINENTAUX DU CALCAIRE (OligOCÈNE-AGÉNIEN SUPÉRIEUR)

Ces fossiles sont représentés par des fragments d'os et des dents isolées. Ils ont tous une couleur noire très prononcée et terne. Ces fossiles ne sont pas roulés. Tous les détails externes sont bien visibles (détails de l'émail). De rares fissures sont visibles. Les cassures franches des os sont nettes et peu ou pas émoussées. Sous la loupe binoculaire, des traces d'argile noire ou de calcaire lacustre blanc sont encore visibles entre les racines ou les plis de l'émail.

\section{LES FOSSILES MARINS DANS LES FACIÈS DU MIOCÈNE MARIN (BURDIGALIEN SUPÉRIEUR-TORTONIEN)}

Dans tous les faciès marins du site paléontologique du Grand Morier, tous les fossiles marins (dents et os) sont roulés et cassés. Leur couleur varie du noir au gris en passant par toutes les teintes de marrons. Tous ces fossiles sont polis et fortement arrondis. Il est parfois très difficile de déterminer certains spécimens. De très nombreux organismes encroûtants marins sont visibles sur ces fossiles (bryozoaires, annélides, balanes). Aucune connexion anatomique n'a été observée même pour les mammifères marins. Toutes ces observations s'appliquent à la fois pour les fossiles remaniés du Crétacé et pour ceux qui se sont déposés pendant le Miocène. Les courants sous-marins miocènes ont désarticulé, cassé et roulé tous ces fossiles.

\section{LES FOSSILES CONTINENTAUX DU CONGLOMÉRAT}

DE LA BASE DU FALUN À BRYOZOAIRES

(LANGHIEN-TORTONIEN INFÉRIEUR)

Au sein de ce conglomérat de base, trois ensembles de fossiles continentaux ressortent avec des aspects bien distincts. Le premier ensemble présente des fossiles peu roulés de couleur noire présentant les mêmes caractéristiques que les fossiles trouvés dans les poches d'argiles noires du calcaire lacustre. Le second ensemble regroupe des dents de couleur grise ainsi que des os de couleur marron très foncé. Ces fossiles sont légèrement patinés et peu roulés. Les dents sont souvent encore positionnées sur leurs mâchoires. Sous la loupe binoculaire, il est possible de voir des grains de quartz coincés entre les racines ou dans les creux des os et des dents. Ces fossiles doivent provenir des sables continentaux de l'Orléanien inférieur. Le troisième ensemble regroupe de nombreuses dents isolées et des os fortement cassés et roulés. Ces fossiles ont des couleurs variant du jaune à l'orange en passant par des couleurs plus ternes (noir à gris foncé). Ces teintes ne sont jamais homogènes sur le même fossile. Tous ces fossiles sont encroûtés par de nombreux animaux marins (bryozoaires, annélides). Ils proviennent d'un sable continental de l'Orléanien supérieur remanié par la transgression du Miocène (Ginsburg \& Mornand 1986).

\section{DISCUSSION}

Six assemblages de vertébrés ont été déterminés: trois assemblages ( $n^{\circ} 1$ à 3$)$ de fossiles de vertébrés en place et/ou faiblement remaniés; et, trois assemblages ( $\mathrm{n}^{\circ} 4$ à 6 ) de fossiles remaniés dans les faluns miocènes. 
ASSEMBLAGE $\mathrm{N}^{\circ} 1$ : LES VERTÉBRÉS CONTINENTAUX EN PLACE DANS L'ARGILE SABLEUSE DE L'ÉOCÈNE

Composition de l'assemblage

Un taxon compose cet assemblage:

- Mammalia: Plagiolophus cf. annectens.

\section{Commentaire}

Le seul fossile de vertébré de cet assemblage provient de l'argile sableuse de l'Éocène. Il correspond à une dent isolée (p4 ou $\mathrm{m} 1$ gauche) de $P$. cf. annectens. Sa détermination avait été faite par L. Ginsburg en 1993 (Gagnaison 2007). Ce dernier avait comparé le fossile du Grand Morier aux fossiles de P. annectens de La Roche-Ragot (Milly-le-Meugon en Anjou, Ginsburg et al. 1978). Les dimensions de la dent du Grand Morier étant légèrement plus petites, Ginsburg l'avait attribué à $P$. cf. annectens. Ce taxon est caractéristique de l'Éocène moyen à supérieur (MP12-20) (Remy 2004). En couplant cette information paléontologique avec les données géologiques, ce premier assemblage confirme que l'argile sableuse fluviatile verte date de l'Éocène moyen à supérieur.

\section{ASSEMBLAGE $\mathrm{N}^{\circ} 2$ : LES VERTÉBRÉS CONTINENTAUX}

EN PLACE DANS LES POCHES D’ARGILE DE DÉCALCIFICATION DU CALCAIRE LACUSTRE DE TOURAINE

(OLIGOCÈNE-AGÉNIEN SUPÉRIEUR)

Composition de l'assemblage

Quatre taxons composent cet assemblage:

- Mammalia: Ronzotherium cf. romani, Phoberocyon hispanicus, Protaceratherium minutum, Paratapirus intermedius.

\section{Commentaire}

Seules des dents de $P$. minutum ont été observées en place dans les poches d'argile noire de décalcification du calcaire lacustre de Touraine sur le site du Grand Morier. Tous les autres fossiles ont été observés dans les collections paléontologiques, mais leur conservation est homogène : la couleur noire des dents, l'absence d'altération mécanique et la présence de petites traces d'argile noire sur les fossiles. Tous les spécimens ont été prélevés dans la partie Nord-Ouest du site du Grand-Morier par différents paléontologues et lors de plusieurs sessions de fouilles. Tous ces fossiles n'ont pas été trouvés ensemble, mais ils doivent provenir de différentes poches de décalcification (avec des contenus paléontologiques d'âges différents: Oligocène ou Miocène inférieur) implantées sur les bordures ou sur le toit de la formation du calcaire lacustre de Touraine.

Les dimensions des dents de P. minutum sont plus petites que celles des fossiles des sites de l'Orléanien inférieur (MN3) de La Guimardière et des Beilleaux (Ginsburg et al. 1981; Gagnaison 2013). Les dents provenant du Grand Morier ont les mêmes dimensions que celles provenant des calcaires lacustres de l'Agénien supérieur (MN2) du site de Selles-surCher (Loir-et-Cher, Ginsburg \& Hugueney 1980 ; Antoine et al. 2000 ; Heissig \& Fejfar 2007). Comme pour le taxon précédent, les fossiles de Paratapirus intermedius (Filhol, 1885) sont identiques à ceux trouvés dans les calcaires agé-

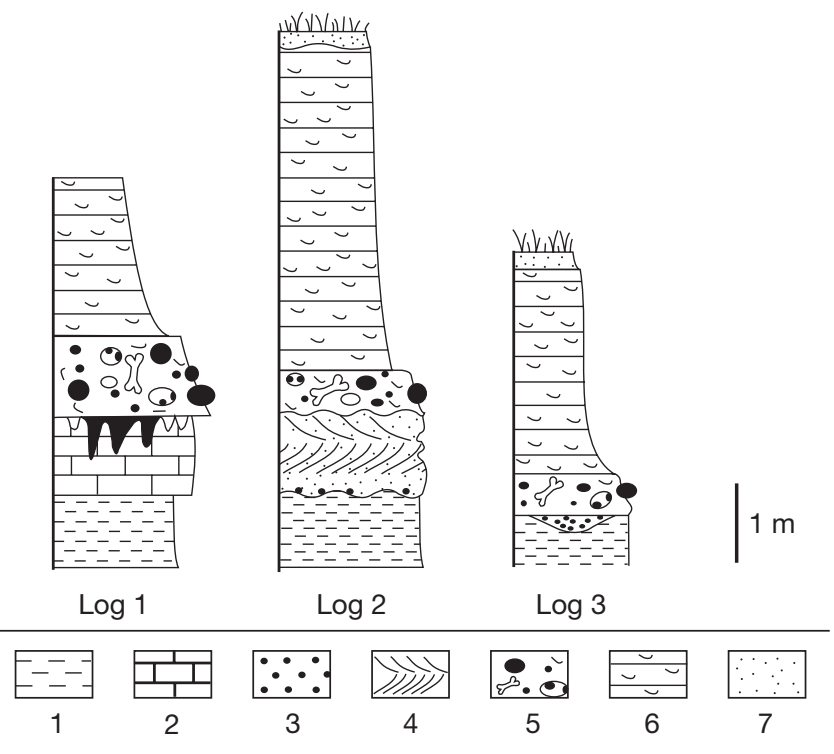

FIG. 5. - Colonnes lithostratigraphiques ( $\log 1, \log 2$ et Log 3) levées sur le site paléontologique du Grand Morier pendant l'exploitation de la falunière. Leurs localisations exactes sont spécifiées sur la carte géologique de PontBoutard (Fig. 2): 1, argile sableuse verte (Éocène); 2, calcaire lacustre avec ses poches d'argile noire de décalcification à son sommet (Oligocène-Agénien supérieur); $\mathbf{3}$, sable continental (Orléanien inférieur); 4, calcarénite à strates oblique (Burdigalien supérieur-Langhien inférieur); $\mathbf{5}$, conglomérat de la base des faluns à bryozoaires (Langhien-Tortonien inférieur); 6, falun à bryozoaires (Tortonien); 7, limons des plateaux chapeautés par la terre végétale actuelle (Quaternaire-Actuel).

niens (MN2) de Selles-sur-Cher (Loir-et-Cher, Ginsburg \& Hugueney 1980 ; Cerdeńo \& Ginsburg 1988; Heissig 1999; Scherler et al. 2011). Ginsburg (2001) cite la présence de Phoberocyon hispanicus Ginsburg \& Morales, 1998 sur le site du Grand Morier. D’après Ginsburg \& Morales (1998), ce mammifère carnivore est typique de l'Agénien supérieur (MN2). La présence du rhinocérotidé Ronzotherium cf. romani Kretzoi, 1945 est également mentionnée sur le site du Grand Morier par Ginsburg (2001). Les molaires supérieures de $R$. cf. romani du site tourangeau sont très proches de celles des argiles oligocènes de Saint-André et Saint-Henri à Marseille (Bouches-du-Rhône, Ménouret \& Guérin 2009). Ronzotherium romani est typique du milieu de l'Oligocène (MP24-29) (Ménouret \& Guérin 2009; Mennecart et al. 2012; Scherler et al. 2013). Cet assemblage $\mathrm{n}^{\circ} 2$ de ces quatre mammifères terrestres marque un âge Oligocène-Agénien supérieur (MP24 à MN2) pour le calcaire lacustre de Touraine (Fahlbusch 1991).

ASSEMBLAGE N³ : LES VERTÉBRÉS MARINS EN PLACE - OU FAIBLEMENT REMANIÉS - DU MIOCÈNE (BURDIGALIEN SUPÉRIEUR À TORTONIEN) DANS LES FACIÈS MARINS MIOCÈNES

Composition de l'assemblage

Vingt-quatre taxons composent cet assemblage:

- Chondrichthyens: Isurus desori, Cosmopolitodus hastalis, Otodus megalodon, Carcharias (Odontaspis) acutissima ou Carcharias (Odontaspis) cuspidata, Carcharias sp., Carcharhinus priscus, Galeocerdo aduncus, Negaprion kraussi, Hemipristis 


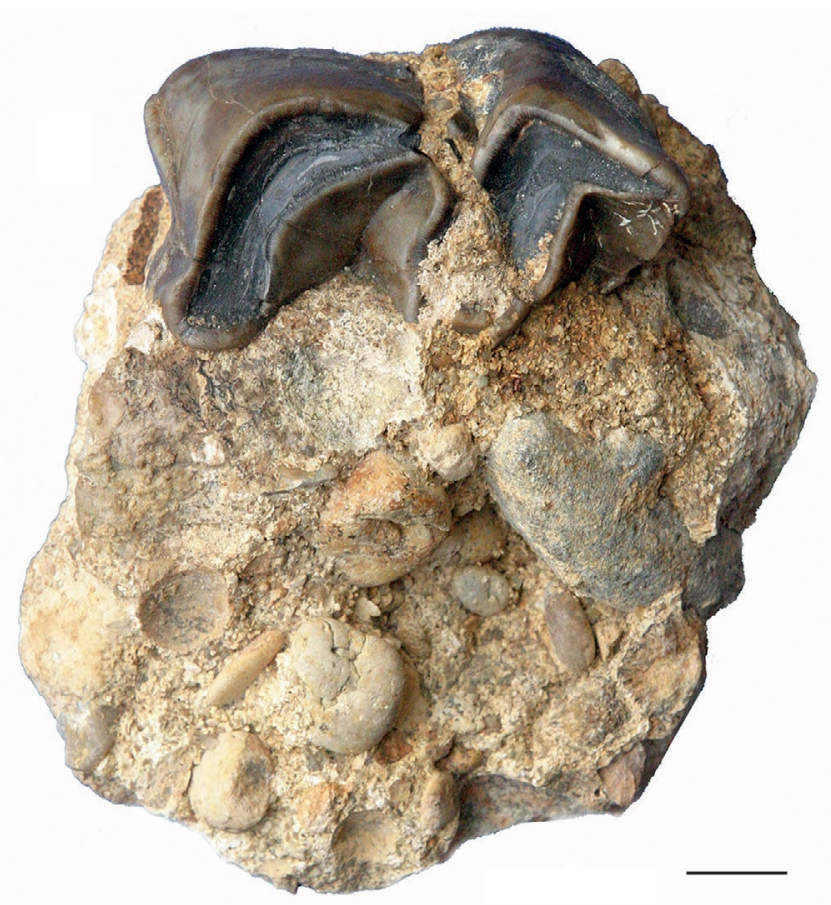

FIG. 6. - m2 g. isolée de Diaceratherium aurelianense (Nouel, 1866) (994PBT-5) reprise dans le conglomérat de base du falun à bryozoaires (Langhien à Tortonien inférieur, assemblage $n^{\circ} 5$ ).

serra, Notorhynchus primigenius, Dasyatis sp., Myliobatidae indet., Aetobatus arcuatus, Pristis aquitanicus;

- Osteichthyes: Sparus cinctus, Sparus aurata, Diplodus jomnitanus, Sphyraena olisiponensis, Labrodon pavimentatum, Tetrodon lecointrae, Trigonodon jugleri;

- Mammalia: Metaxytherium sp., Orycterocetus crocodilinus, Cetacea indet.

\section{Commentaire}

L'ensemble des fossiles provient des trois faciès marins du Miocène du Grand Morier. L'association paléoichtyologique est identique à celles connues depuis de nombreuses années dans les faluns du golfe ligérien: de l'Anjou-Touraine (Mornand 1978; Pouit 2009), du Blésois (Mornand 1978), de la Vendée (Leriche 1957) et de la Bretagne (Leriche 1957). Cette association de chondrichthens et des osteichtyes littoraux est datée du Miocène Moyen à Supérieur : Burdigalien supérieur à Tortonien (Courville \& Bongrain 2003). Les fossiles de mammifères marins sont en très mauvais état: des fragments de côtes et de vertèbres pouvant se rapporter au sirénien Metaxytherium sp. ou à un cétacé indéterminable et des dents allongées d'Orycterocetus crocodilinus Cope, 1868. À partir des différents travaux de Ginsburg \& Janvier $(1971,1999)$ et de Bianucci et al. (2004), il est possible d'en déduire que ce cortège de mammifères marins date du Miocène Moyen-Supérieur : Langhien à Tortonien. Cet assemblage $n^{\circ} 3$ est cohérent avec des faunes de poissons et de mammifères marins contemporains à la mise en place des faciès marins (la calcarénite, le conglomérat et les faluns à bryozoaires) du Miocène (Burdi- galien supérieur à Tortonien) en contexte de mer subtropicale à tempérée chaude peu profonde (Gagnaison 2013).

\section{ASSEMBLAGE N² 4 : LES VERTÉBRÉS DU CRÉTACÉ SUPÉRIEUR REMANIÉS DANS LE CONGLOMÉRAT MIOCÈNE (LANGHIEN- TORTONIEN INFÉRIEUR) \\ Composition de l'assemblage \\ Trois taxons composent cet assemblage: \\ - Chondrichthyens: Squalicorax sp., Cretolamna sp., Ptycho- dus sp.}

\section{Commentaire}

Ces fossiles très roulés et cassés proviennent tous des graviers ou du conglomérat de base des faciès marins du Miocène. Ces chondrichtyens sont classiques des faciès carbonatés du Crétacé supérieur de l'Anjou-Touraine (Lecointre 1947 ; Biddle 1993). Cette faune est même très abondante dans le « falun de Continvoir» du Turonien supérieur de l'ancienne carrière de Pont-Boutard située à moins de 600 mètres au nord du site du Grand Morier (Cappetta 2004). Il est facile d'imaginer que les faciès du Turonien supérieur de Pont-Boutard ont été en partie érodés par la transgression miocène. Ainsi les fossiles crétacés se sont retrouvés incorporés directement dans les sédiments miocènes (Gobé et al. 1980).

ASSEMBLAGE N ${ }^{\circ}$ : LES VERTÉBRÉS CONTINENTAUX DES SABLES FLUVIATILES (ORLÉANIEN INFÉRIEUR) REMANIÉS DANS LE CONGLOMÉRAT MIOCÈNE (BURDIGALIEN SUPÉRIEUR-TORTONIEN) Composition de l'assemblage

31 taxons composent cet assemblage:

- Sauropsida: Ptychogaster sp., Testudo sp., Trionyx sp., Diplocynodon sp.;

- Mammalia: Lagopsis sp., Eucricetodon infralactorensis, Steneofiber depereti, Amphicyon (Megamphicyon) lathanicus, Cynelos helbingi, Cynelos schlosseri, Haplocyonoides mordax, Ballusia hareni, Phoberocyon aurelianensis, Phoberocyon dehmi, Plithocyon bruneti, Hemicyon gargan, Martes laevidens, Palaeogale hyaenoides, Plesiogale angustifrons, Pseudaelurus turnauensis, Anchitherium aurelianense, Protaceratherium minutum, Diaceratherium aurelianense, Aureliachoerus aurelianensis, Xenohyus venitor, Brachyodus onoideus, Brachyodus intermedius, Procervulus dichotomus, Heterocemas sp., Ligeromeryx praestans, Andegameryx andegaviensis.

\section{Commentaire}

Des fossiles de cet assemblage ont été trouvés dans les trois faciès du Miocène marin, mais la majeure partie (75\% de l'ensemble des fossiles étudiés) provient du conglomérat miocène (Langhien-Tortonien inférieur, Fig. 6). Ces taxons sont déjà tous connus dans les sables continentaux de l'Orléanien inférieur (MN3) des sites de l'Anjou-Touraine tels que La Guimardière (Maine-et-Loire), Les Beilleaux (Indre-et-Loire) et Pontigné (Maine-et-Loire, Ginsburg \& Bonneau 1995; Ginsburg 2001; Gagnaison 2013). Même si cet assemblage est remanié, il constitue une association faunique typique du début de l'Orléanien (MN3; Ginsburg 1989a; Mein 1999). 
A
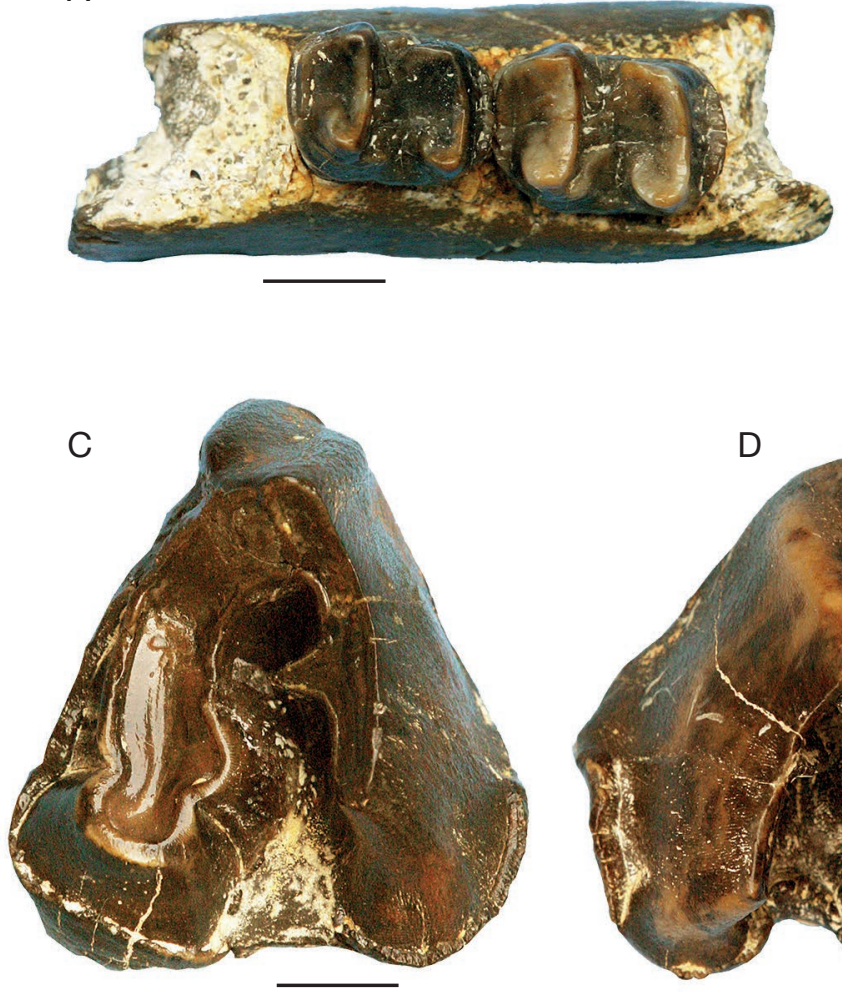

$\mathrm{F}$
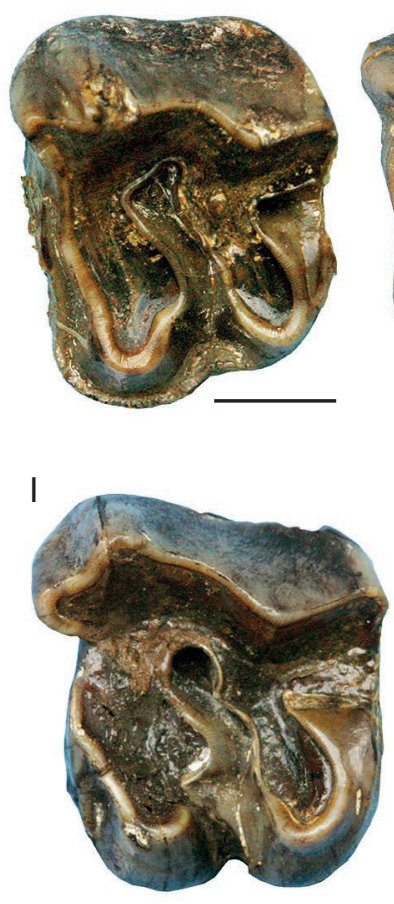

G

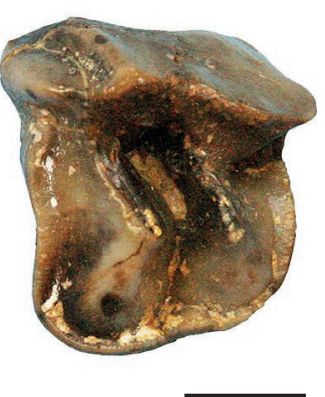

J

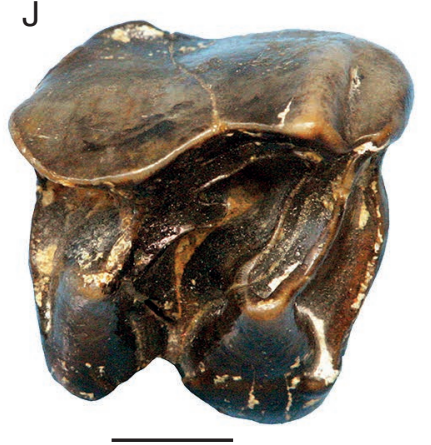

西

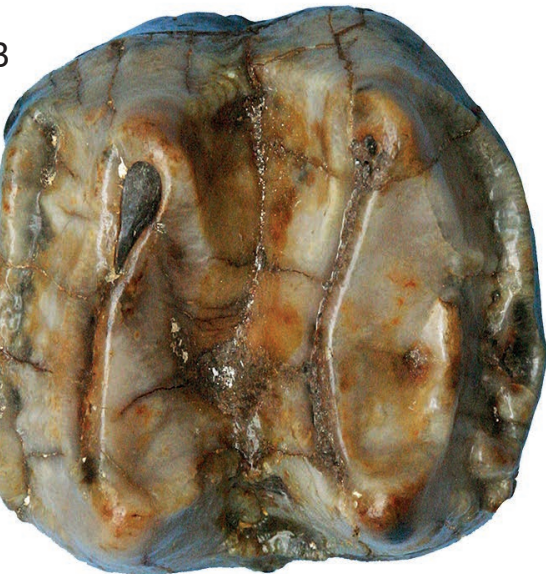

$\mathrm{E}$

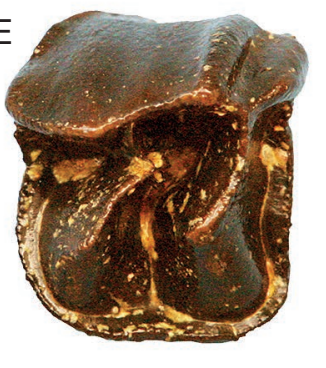

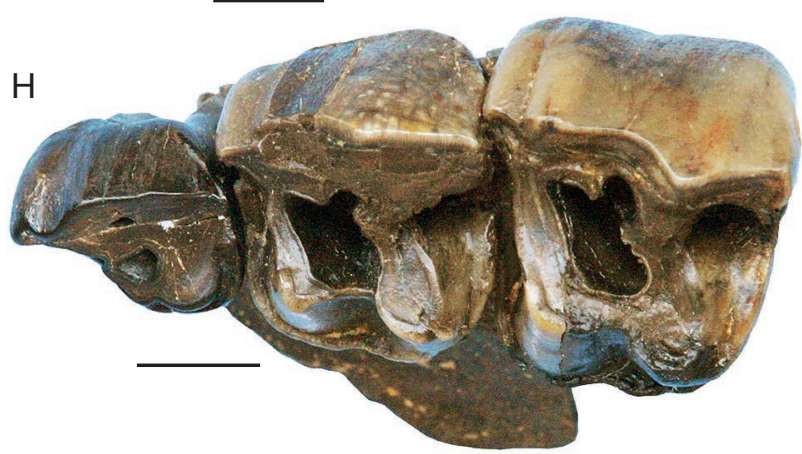

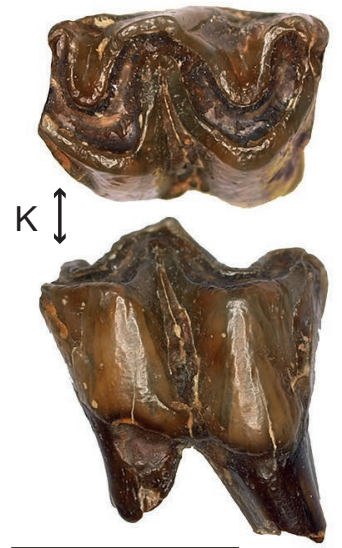

FIG. 7. - Exemples de fossiles de mammifères cénozoïques du Grand Morier (Pont-Boutard, Indre-et-Loire, France) provenant de la collection Quétin du Musée du Savignéen (Savigné-sur-Lathan, Indre-et-Loire, France): A, Paratapirus intermedius (Filhol, 1885) (assemblage ${ }^{\circ} 2$ ), hémimandibule d. portant m1-2 (2012-PBT-4); B, Prodeinotherium bavaricum Von Meyer, 1831 (assemblage $n^{\circ} 6$ ), M2 d. isolée (2012-PBT-289); C, D, Diaceratherium aurelianense (Nouel, 1866) (assemblage $\mathrm{n}^{\circ}$ 5); C, M3 g. isolée (2012-PBT-51); D, M3 d. isolée (2012-PBT-49); E-K, Protaceratherium minutum (Cuvier, 1822-1824) (assemblages n² et n5); E, P3 d. isolée (2012-PBT-137); F, P4 g. isolée (2012-PBT-139); G, P3 g. isolée (2012-PBT-136); H, montage d'une série dentaire portant P1-3 g. (2012-PBT-135); I, M1 g. isolée (2012-PBT-128); J, M2 d. isolée (2012-PBT-130); K, Plagiolophus cf. annectens (Owen, 1847) (assemblage n¹), p4 ou m1 g. isolée (LB-PBT-1). Échelles: 1 cm. 
ASSEMBLAGE N ${ }^{\circ} 6$ : LES VERTÉBRÉS CONTINENTAUX DE L'ORLÉANIEN SUPÉRIEUR REMANIÉS DANS LES FACIÈS MARINS DU MiocÈnE (BURDIGALIEN SUPÉRIEUR-TORTONIEN) Composition de l'assemblage

49 taxons composent cet assemblage:

- Sauropsida: Chelydropsis sp., Ptychogaster sp., Testudo promarginata, Testudo sp., Trionyx sp., Lacertidae indet., Boïdae indet., Colubridae indet., Viperidae indet., Diplocynodon styriacus, Diplocynodon sp., Tomistoma cf. lusitanica, Gavialis sp., Incertae sedis;

- Mammalia : Proscapanus sansaniensis, Lagopsis sp., Steneofiber depereti, Anchitheriomys wiedemanni, Pliopithecus piveteaui, Hyainailouros sulzeri, Amphicyon giganteus, Agnotherium grivensis, Pseudocyon sansaniensis, Pseudarctos bavaricus, Hemicyon sansaniensis, Trocharion albanense, Protictitherium gaillardi, Pseudaelurus romievensis, Pseudaelurus lorteti, Pseudaelurus turnauensis, Prodeinotherium bavaricum, Gomphotherium angustidens, Zygolophodon turicensis, Anchitherium aurelianense, Anisodon grande, Brachypotherium brachypus, Plesiaceratherium lumiarense, Prosantorhinus germanicus, Prosantorhinus douvillei, Lartetotherium sansaniense, Aureliachoerus aurelianensis, Bunolistriodon lockharti, Hyotherium soemmeringi, Dorcatherium guntianum, Dorcatherium crassum, Amphimoschus pontileviensis, Dicrocerus elegans, Lagomeryx sp., Palaeomeryx bojani.

\section{Commentaire}

Tous les fossiles proviennent des faciès marins du Miocène. Ils sont tous remaniés d'au moins un faciès continental (un sable fluviatile ou un calcaire lacustre) inconnu à l'affleurement. Tous ces taxons sont déjà cités à plusieurs reprises dans les nombreux gisements miocènes de l'Orléanien moyen-supérieur (MN4 à MN5) du golfe ligérien: les falunières de l'Anjou-Touraine (Ginsburg 1990), les sablières de l'Orléanais (Mayet 1908 ; Cerdeño 1993; Ginsburg 2002) ou encore les falunières du Blésois (Augé et al. 2002; Gagnaison et al. 2006). Aucune nouveauté n'est à noter dans cette série de taxons que Ginsburg (2001) avait proposé de regrouper dans la biozone de l'Orléanien supérieur (MN5). Même s'il est délicat de faire un tri précis dans tout cet assemblage $n^{\circ} 6$, la proposition biostratigraphique de Ginsburg s'accorde bien avec le tableau de synthèse pour les mammifères terrestres du site du Grand Morier (Fig. 8). Donc, l'assemblage $n^{\circ} 6$ peut se rapporter à l'Orléanien supérieur (MN5) (Steininger 1999; Agusti et al. 2001).

\section{CONCLUSIONS}

Grâce aux observations faites directement sur le site paléontologique du Grand Morier ainsi que dans les collections paléontologiques il a été possible de faire ressortir deux données importantes: un contexte géologique complexe (stratigraphie et tectonique) et six assemblages de vertébrés bien distincts (un du Crétacé supérieur, un de l'Éocène, un de l'Oligocène-Miocène inférieur et trois du Miocène).

\section{CONTEXTE GÉOLOGIQUE PARTICULIER}

Les affleurements du Cénozoïque sont nombreux et diversifiés en Anjou-Touraine. Le Crétacé est recouvert par des formations continentales paléogènes et marines néogènes, mais, il est très rare d'avoir une série stratigraphique complète de l'ensemble des formations cénozoïques sur un même site paléontologique. Il est d'autant plus exceptionnel d'observer des fossiles de vertébrés dans chacune des différentes couches géologiques. Même si plusieurs faciès ne sont représentés que par des lambeaux, Le Grand Morier est le seul site connu pour le moment - en Anjou-Touraine présentant la succession stratigraphique complète de tous les faciès cénozoïques depuis les "perrons de Touraine" (Paléocène-Éocène) au sable à graviers (Miocène terminal-Pliocène). Dans le cadre de l'inventaire national des sites géologiques importants à conserver en France (projet lancé par le MNHN), une fiche a été rédigée sur Le Grand Morier pour le dossier concernant le département de l'Indre-et-Loire. Ce travail d'inventaire met surtout en valeur l'importance des diversités stratigraphique et paléontologique de cette ancienne falunière.

Même si de nombreuses publications citent la réactivation des failles hercyniennes au cours du Cénozoïque en AnjouTouraine, le contexte tectonique du graben de Pont-Boutard reste très particulier. De même, les transgressions miocènes sont canalisées dans le bassin miocène de Savigné-sur-Lathan/ Noyant-sous-le-Lude par une série de failles verticales à composante normale. En couplant les informations stratigraphiques et tectoniques, il apparaît clairement que la sédimentation cénozoïque a été canalisée et contrainte par le mouvement des failles du graben de Pont-Boutard. Dans le détail, la mise en place des formations continentales (les dépôts fluviatiles et lacustres) du milieu du Cénozoïque (Éocène au Miocène inférieur) est induite par à la formation d'une cuvette morphologique directement liée à l'activation des failles passant par le hameau de Pont-Boutard et par le lieu-dit du Grand Morier pendant l'orogenèse pyrénéenne. La tectonique alpine (Miocène) va accentuer l'approfondissement du graben de Pont-Boutard permettant à la fois la sauvegarde d'une grande partie de la série sédimentaire anté-faluns miocènes et la canalisation des sédiments marins miocènes progradants du bassin de Savigné-sur-Lathan/Noyant-sous-le-Lude vers le bassin de Paulmy dans la Touraine du Sud.

\section{CONTEXTE PALÉONTOLOGIQUE}

Même si la présence d'un fossile de $P$. cf. annectens dans l'argile sableuse verte de l'Éocène est exceptionnelle dans le site du Grand Morier (assemblage $n^{\circ} 1$ de l'Éocène moyen à supérieur, MP12 à MP20), elle n'est cependant pas surprenante en Anjou-Touraine. Ce taxon a déjà été cité à deux reprises dans la bibliographie sur les mammiferes de l'Anjou-Touraine: une série de fossiles provenant des sables verts de l'Éocène supérieur du site paléontologique de La Roche-Ragot (Millyle-Meugon en Anjou) ainsi qu'une molaire isolée et remaniée dans les faluns miocènes de l'Anjou-Touraine (la localité exacte de cette découverte n'est pas précisée).

Les quatre mammifères terrestres (Ronzotherium cf. romani Kretzoi, 1945, d'âge oligocène; Phoberocyon hispanicus Gins- 


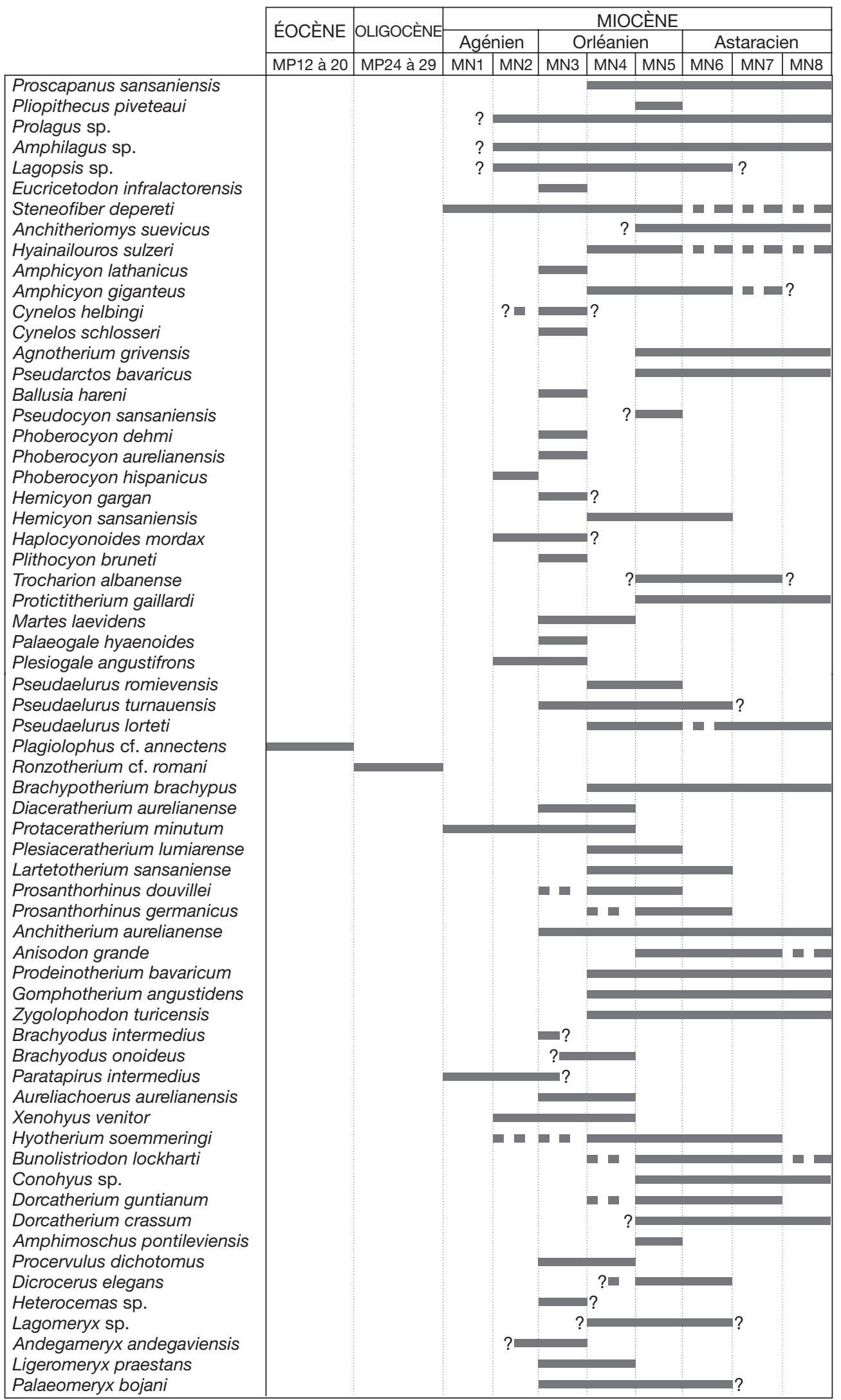

FiG. 8. - Répartition biostratigraphique européenne des mammifères terrestres cénozoïques représentés sur le site paléontologique du Grand Morier (PontBoutard, Indre-et-Loire, France). Ce tableau a été principalement réalisé à l'aide des publications suivantes: Ginsburg (1999); Antoine et al. (2000); Ménouret \& Guérin (2009); Gagnaison (2013); Scherler et al. (2013). 
burg \& Morales, 1998, Protaceratherium minutum (Cuvier, 1822-1824) et Paratapirus intermedius Filhol, 1885 du Miocène basal) provenant des calcaires lacustres sous-jacents aux faluns marins miocènes (assemblage $\mathrm{n}^{\circ} 2$ ) apportent un éclairage nouveau pour la biostratigraphie de l'Anjou-Touraine. Ce calcaire lacustre de Touraine a toujours été daté de l'Éocène supérieur à l'Oligocène par des observations faites sur les associations de gastéropodes pulmonés. La présence de mammiferes terrestres identiques à ceux déjà observés sur le site paléontologique de Selles-sur-Cher (Loir-et-Cher) dans le faciès lacustre du site paléontologique du Grand Morier permet de proposer une datation plus récente pour le calcaire lacustre de Touraine: Oligocène-Agénien supérieur, MP24 à MN2.

L'assemblage $\mathrm{n}^{\circ} 3$ est constitué exclusivement de vertébrés marins (poissons et mammifères) miocènes déjà bien connus. De nombreuses publications ont déjà présenté ces listes fauniques typiques des faluns marins de la gouttière ligérienne dont l'âge englobe une grande partie du Miocène: Burdigalien supérieur au Tortonien. La faune marine du site du Grand Morier est comparable à celle des sites de La Morfassière (Indre-et-Loire) ou de Pelmer (Maine-et-Loire).

Les rares fossiles du Crétacé supérieur (assemblage $\mathrm{n}^{\circ}$ 4) proviennent du remaniement des faciès du Turonien supérieur - situés à proximité du site du Grand Morier - dans les faluns miocènes. Ces fossiles sont en trop mauvais état pour en tirer d'autres informations.

Les faunes de vertébrés de l'Orléanien inférieur (MN3) (assemblage $\mathrm{n}^{\circ} 5$ ) ont subi un très faible remaniement comme le prouvent leur bonne conservation et la présence d'une lentille de sable fluviatile de l'Orléanien inférieur dans la partie orientale du site paléontologique du Grand Morier. Tous les taxons de cet assemblage sont déjà connus dans les sables fluviatiles de l'Orléanien inférieur (MN3) des sites paléontologiques régionaux suivants: Mauvières (Indre-et-Loire), La Brosse (Maine-et-Loire), Chitenay (Loir-et-Cher), Les Beilleaux (Indre-et-Loire), Pontigné (Maine-et-Loire) et La Guimardière (Maine-et-Loire).

Les fossiles de l'Orléanien supérieur (MN5) (assemblage n6) du Grand Morier sont tous remaniés d'un faciès continental inconnu à l'affleurement. Malgré l'état des fossiles - parfois mauvais - il est possible d'en déduire que cet ensemble est cohérent. Toutefois, il serait très délicat de faire des comparaisons avec des faunes en place des gisements paléontologiques du Blésois ou de l'Orléanais. Les taxons de l'assemblage $n^{\circ} 6$ s'intègrent parfaitement dans la liste de «faunes contemporaines des faluns » définie par Ginsburg (2001) pour les gisements miocènes (MN5) du bassin de Savigné-sur-Lathan/ Noyant-sous-le-Lude.

\section{Remerciements}

Ce travail n'aurait jamais pu être réalisé sans l'aide de C. Andrin \& A. Mathivon (étudiants en géologie à l'Institut polytechnique UniLaSalle à Beauvais), R. Rateau, B. Cossard, J.-P. Guillon et S. Xerri. Qu'ils soient tous remerciés. Merci aussi aux musées (J.-C. Gagnaison pour le Musée du Savignéen, L. Ginsburg pour le MNHN et P. Watelet pour le
MHN Tours) et à tous les paléontologues amateurs de m'avoir ouvert les tiroirs de leurs collections. Un grand merci à la famille Quétin qui a récolté de nombreux fossiles du Grand Morier conservés dans le Musée du Savignéen (Annexe 2). Cette collection n'aurait jamais pu rentrer dans ce musée régional sans les soutiens de P. Janvier (MNHN) et de la DRAC Centre. Merci à P.-O. Antoine (Institut des Sciences de l'Évolution de Montpellier) et B. Mennecart (Naturhistorisches Museum Basel) d'avoir relu ce travail.

\section{RÉFÉRENCES}

Aguilar J.-P., Legendre S. \& Michaux J. 1997. — BiochroM'97: Biochronologie mammalienne du Cénozoïque en Europe et domaines reliés. Mémoires et Travaux E.P.H.E., Institut universitaire de Montpellier 21: 1-817.

Agusti J., Cabrera L., Garcès M., Krijgsman W., Oms O. \& Parès J. M. 2001. - A calibrated mammal scale for the Neogene of Western Europe. State of the art. Earth-Science Reviews 52: 247-260. https://doi.org/10.1016/S0012-8252(00)00025-8

AlCAYDÉ G. 1975. - Carte géologique de la France au 1/50 000: feuille de Chinon. Bureau de Recherches Géologiques et Minières, Orléans 486: 1-20, 1 carte.

AlCAYDÉ G. \& RASPlus L. 1971. — Carte géologique de la France au 1/50 000: feuille de Langeais. Bureau de Recherches géologiques et minières 487: 1-12, 1 carte.

Alcaydé G., Gigout M., CADET J.-P., Lorenz J., Brosse R., DebrandPASSARD S., LORENZ C., RAMPNOUX J.-P. \& RASPLUS L. 1976. — Val de Loire: Anjou - Touraine - Berry. Masson, Paris: 1-191 (Guides géologiques régionaux).

ANDRIN C. \& MATHIVON A. 2015. - Cartographie géologique du graben de Pont-Boutard (37 France). Mémoire d'Ingénieur géologue, Institut Polytechnique LaSalle Beauvais, Beauvais 722: 1-65, inédit.

Antoine P.-O. \& BeCKer D. 2013. - A brief review of Agenian rhinocerotids in Western Europe. Swiss Journal of Geosciences 106: 135-146. https://doi.org/10.1007/s00015-013-0126-8

Antoine P.-O., Bulot C. \& Ginsburg L. 2000. — Les rhinocérotidés (Mammalia, Perissodactyla) de l'Orléanien des bassins de la Garonne et de la Loire (France): intérêt biostratigraphique. Comptes Rendus de l'Académie des Sciences. Series IIA. Sciences de la Terre et des Planètes 330 (8): 571-576. http://doi.org/10.1016/S1251-8050(00)00174-9

AnTunes M. T. \& Ginsburg L. 1989. - Les Crocodiliens des faluns miocènes de l'Anjou. Bulletin du Muséum national d'Histoire naturelle, 4e série, section C, Sciences de la terre, paléontologie, géologie, minéralogie 11 (2): 79-99.

Augé M., Ginsburg L., Lapparent de Broin F. de, Makinsky M., Mourer C., Pouit D. \& Sen S. 2002. - Les vertébrés du Miocène moyen de Contres (Loir-et-Cher, France). Revue de Paléobiologie 21 (2): 819-852.

BianUCCi G., LANDini W. \& VAROla A. 2004. - First discovery of the Miocene northern Atlantic sperm whale Orycterocetus in the Mediterranean. Geobios 37: 569-573. https://doi.org/10.1016/j. geobios.2003.05.004

BIDDLE J.-P. 1993 - Découverte d'une faunule d'âge crétacé dans le Miocène moyen du Sud-Ouest du Bassin de Paris. Cossmanniana H.S. 2: 39-42.

BOUCHET E. 2009. —Étude sédimentologique des faluns miocènes de l'Anjou-Touraine: Bassin de Savigné-sur-Lathan/Noyant. Mémoire d'Ingénieur Géologue, Institut Polytechnique LaSalle Beauvais, Beauvais 451: 1-90, inédit.

Bouchet E., Gagnaison C., Sterbik N. \& Rateau R. 2010. New paleontological and sedimentological data on the Miocene basin of Savigné-sur-Lathan/Noyant-sous-le-Lude (Indre-et-Loire/ Maine-et-Loire, France). Congrès Strati2010 du 30/08 au 02/09 2010, UPMC, Paris 6, livret des résumés: 39-41. 
Bruijn H. De, Daams R., Daxner-HÖck G., Fahlbusch V., Ginsburg L., Mein P. \& Morales J. 1992. - Report of the RCMNS working group on fossil mammals, Reisensburg 1990. Newsletters on Stratigraphy 26 (2-3): 65-118. https://dx.doi. org/10.1127/nos/26/1992/65

Buge E. 1948. — Les bryozoaires du Savignéen (Helvétien) de Touraine: essai de paléogéographie du Néogène de l'Ouest de la France. Muséum national d'Histoire naturelle, Paris, 31 p. (Mémoires du Muséum national d'Histoire naturelle - Nouvelle série; 27 [3]).

CABIDOCHE M. \& Riera R. 2013. — Étude sédimentaire des sables continentaux du Burdigalien en Touraine. Mémoire d'Ingénieur Géologue, Institut Polytechnique LaSalle Beauvais, Beauvais 618 : $1-67$, inédit.

Cappetta H. 2004. - Sur une nouvelle espèce de Ptychotrygon (Neoselachii: Rajiformes) du Turonien supérieur de Touraine, France. Neues Jahrbuch für Geologie und Paläontologie, Monatshefte (1): 41-52.

CERDEÑO E. 1993. - Étude sur Diaceratherium aurelianense et Brachypotherium brachypus (Rhinocerotidae, Mammalia) du Miocène moyen de France. Bulletin du Muséum national d'Histoire naturelle, 4 e série, section $C$, Sciences de la terre, paléontologie, géologie, minéralogie 15 (1-4): 25-77.

Cerdeño E. \& Ginsburg L. 1988. - Les Tapiridae (Perissodactyla, Mammalia) de l'Oligocène et du Miocène inférieur européens. Annales de Paléontologie (Vertébrés-Invertébrés) 74: 71-96.

Courville P. \& Bongrain M. 2003. - Les Pectinidae miocènes des faluns (Ouest de la France). Intérêts biostratigraphiques des associations. Annales de Paléontologie 89: 125-151. https://doi. org/10.1016/S0753-3969(03)00022-3

Debelmas J. 1974. - Géologie de la France. Édition Doin, Paris, volumes $1 \& 2: 1-540$

Desforges R. \& Merlu N. 2010. — Étude sédimentologique des formations crétacées des vallées de la Loire et du Cher. Étude pétrographique des silex de la Roche-Cotard (Langeais, Indreet-Loire). Mémoire d'Ingénieur Géologue, Institut Polytechnique LaSalle Beauvais, Beauvais 498: 1-87, inédit.

Dourlat E. \& EMERIAU G. 2012. — Étude sédimentologique des argiles kaoliniques blanches à spongiaires du Crétacé de Touraine. Étude historique de l'exploitation et de l'utilisation des argiles kaoliniques blanches de Langeais et Paulmy (Indre-et-Loire). Mémoire d'Ingénieur Géologue, Institut Polytechnique LaSalle Beauvais, Beauvais 586: 1-110, inédit.

FAHLBUSCH V. 1991. — The meaning of MN-zonation. Considerations for a subdivision of the European Tertiary. Newsletters on Stratigraphy 5: 160-167. https://doi.org/10.1127/nos/24/1991/159

Gagnaison C. 2007. - Une dent de Plagiolophus remaniée dans les faluns miocènes de Pont-Boutard (37, France). Bulletin de la Société d'Études scientifiques de l'Anjou 21: 115-118.

GaGnaison C. 2012. - Des bernard-l'hermites dans les faluns miocènes de Channay-sur-Lathan (Indre-et-Loire, France). Cossmanniana 14: 67-72.

GAGNAISON C. 2013. — Les assemblages de vertébrés dans deux sites paléontologiques du bassin miocène de Savigné-sur-Lathan/ Noyant-sous-le-Lude: La Guimardière et Pelmer (Maine-et-Loire, France). Geodiversitas 35 (1): 67-103. https://doi.org/10.5252/ g2013n1a5

GaGnaison C., Gillet P.-A. \& FuCCI D. 2004. —Étude taphonomique du site Miocène de La Guimardière (Maine-et-Loire, France). Mémoire de la Société d'Études scientifiques de l'Anjou 16: 1-95.

Gagnaison C., Castillo L., Grugier O., Renou J.-C. \& FucCI D. 2006. - Étude paléoécologique du site miocène de Contres (41, France) : diversité animale et taphonomie. Symbioses, nouvelle série 16: 19-25.

GINSBURG L. 1989a. - The faunas and stratigraphical subdivisions of the Orleanian in the Loire basin (France), in LINDSAY E. H., Fahlbusch V. \& Mein P. (eds), European Neogene Mammal Chronology. NATO ASI Series 180, Plenum Press, New York; London: 157-176.
GiNSBURG L. 1989b. — Les mammifères des sables du Miocène inférieur des Beilleaux à Savigné-sur-Lathan (Indre-et-Loire). Bulletin de la Société d'Études scientifiques d'Anjou 13: 35-52.

GINSBURG L. 1990. — Les quatre faunes de Mammifères miocènes des faluns du synclinal d'Esvres (Val-de-Loire, France). Comptes rendus des séances de l'Académie des sciences, Sér. II, Mécanique, physique, chimie, sciences de l'univers, sciences de la terre 310 (2): 89-93.

Ginsburg L. 1999. - Order Carnivora, in Rössner G. E. \& HeIssig K. (éds), The Miocene Land Mammals of Europe. F. Pfeil, München: 109-148.

GiNSBURG L. 2001. - Les faunes de mammifères terrestres du Miocène moyen des Faluns du bassin de Savigné-sur-Lathan (France). Geodiversitas 23 (3): 381-394.

GINSBURG L. 2002. — Les carnivores fossiles des sables de l'Orléanais. Annales de Paléontologie 88: 115-146. https://doi.org/10.1016/ S0753-3969(02)01042-X

GinsBurg L. \& BONNEAU M. 1995. — La succession des faunes de mammiferes miocènes de Pontigné (Maine-et-Loire, France). Bulletin du Muséum national d'Histoire naturelle, 4e série, section C, Sciences de la terre, paléontologie, géologie, minéralogie 16 (2-4): 313-328.

GinsBuRG L. \& HuguENEY M. 1980. — La faune des mammiferes du Miocène inférieur de Selles-sur-Cher (Loir-et-Cher). Bulletin du Muséum national d'Histoire naturelle, 4e série, section C, Sciences de la terre, paléontologie, géologie, minéralogie 2 (3): 271-276.

GINSBURG L. \& JANVIER P. 1971. — Les mammiferes marins des faluns miocènes de la Touraine et de l'Anjou. Bulletin du Muséum national d'Histoire naturelle, 3e série, Sciences de la Terre, 22 (6): 161-195.

Ginsburg L. \& Janvier P. 1999. — Les phoques (Phocidae, Pinnipedia, Carnivora, Mammalia) des faluns miocènes de l'Anjou. Bulletin de la Société des Sciences naturelles de l'Ouest de la France, nouvelle série 21: 169-178.

GinSBURG L. \& MorNAND J. 1986. — Les restes de mammifères des faluns de l'Anjou-Touraine. Mémoire de la Société d'Études scientifiques de l'Anjou 6: 1-73.

GinsBurg L. \& Morales J. 1998. — Les Hemicyoninae (Ursidae, Carnivora, Mammalia) et les formes apparentées du Miocène inférieur et moyen d'Europe occidental. Annales de Paléontologie 84 (1): 71-123. https://doi.org/10.1016/S0753-3969(98)80003-7

GinsBURG L., HUIN J. \& LOCHER J.-P. 1981. — Les Rhinocerotidae (Perissodactyla, Mammalia) du Miocène inférieur des Beilleaux à Savigné-sur-Lathan (Indre-et-Loire). Bulletin du Muséum national d'Histoire naturelle, 4 e série, section $C$, Sciences de la terre, paléontologie, géologie, minéralogie 3 (4): 345-361.

Ginsburg L., Janvier P., Jehenne Y. \& Yvard J.-C. 1978. — Un gîte à Mammifères d'âge éocène supérieur en Anjou. Comptesrendus sommaires de la Société géologique de France 4: 185-186.

Ginsburg L., Cheneval J., Janvier P., Pouit D. \& Sen S. 2000. Les vertébrés des sables continentaux d'âge orléanien inférieur (MN3) de Mauvières à Marcilly-sur-Maulne (Indre-et-Loire), La Brosse à Meigné-le-Vicomte (Maine-et-Loire) et Chitenay (Loir-et-Cher). Geodiversitas 22 (4): 597-631.

Gobé J.-F., Mornand J. \& Pouit D. 1980. — Les restes de reptiles des faluns de l'Anjou-Touraine (et supplément Poissons). Mémoire de Société d'Études scientifiques de l'Anjou 5: 1-30.

HeISSIG K. 1999. — Family Tapiridae, in Rössner G. E. \& HeIsSIG K. (éds), The Miocene Land Mammals of Europe. F. Pfeil, München: 171-174.

Heissig K. \& FejFAr O. 2007. — Die fossilen nashörner (Mammalia, Rhinocerotidae) aus dem untermiozän von Tuchořice in norwestböhmen. Acta Musei Nationalis Pragae, series B, 63 (1): 19-64.

Hom L. \& Moulin M. 2012. — Les séries sédimentaires du Paléogène-Miocène supérieur du bassin de Noyant-sous-le-Lude/ Savigné-sur-Lathan (Touraine). Mémoire d'Ingénieur géologue, Institut Polytechnique LaSalle Beauvais, Beauvais 590: 1-74, inédit. LECOINTRE G. 1930. - Révision de la feuille de Tours au 1/80 000. Bulletin de la Société géologique de France 187: 131-133.

LeCointre G. 1947. - Géologie régionale de la France. 4: La Touraine. Actualités scientifiques et industrielles 1027: 1-240. 
LERICHE M. 1957. — Les poissons des faluns néogènes de l'Ouest de la France (Bretagne et Cotentin, Anjou, Touraine). Mémoires de la Société géologique de France, nouvelle série, t. XXXVI, 81: 1-64. MAYET L. 1908. - Étude des mammiferes miocènes des sables de l'Orléanais et des faluns de la Touraine. Annales de l'Université de Lyon, nouvelle série 24 (1): 1-336. https://doi.org/10.5962/bhl.title.52982

MeIN P. 1999. - European Miocene Mammal Biochronology, in Rössner G. E. \& HeISsIg K. (éds), The Miocene Land Mammals of Europe. F. Pfeil, München: 25-38.

Mennecart B., Scherler L., Hiard F., Becker D. \& Berger J.-P. 2012. - Large mammals from Rickenbach (Switzerland, Reference-Locality MP29, Late Oligocene): biostratigraphic and paleoenvironmental implications. Swiss Journal of Palaeontology 131: 161-181. https://doi.org/10.1007/s13358-011-0031-6

MénOURET B. \& GUÉRIN C. 2009. - Diaceratherium massiliae nov. sp. des argiles oligocènes de Saint-André et Saint-Henri à Marseille et de Les Milles près d'Aix-en-Provence (SE de la France), premier grand Rhinocerotidae brachypode européen. Geobios 42: 293-327. https://doi.org/10.1016/j.geobios.2008.10.009

MoRNAND J. 1978. - Les restes de poissons des faluns de l'AnjouTouraine. Mémoire de la Société d'Études scientifiques de l'Anjou 3: 1-23.

PouiT D. 2009. — Les poissons des faluns miocènes de la collection Hartmann du Musée du Savignéen. Symbioses, nouvelle série 23: 12-18.

Remy J. A. 2004. — Le genre Plagiolophus (Palaeotheriidae, Perissodactyla, mammalia): révision systématique, morphologie et histologie dentaires, anatomie crânienne, essai d'interprétation fonctionnelle. Palaeovertebrata 33 (1-4): 17-281.

Scherler L., Becker D. \& Berger J.-P. 2011. — Tapiridae (Perissodactyla, Mammalia) of the Swiss Molasse Basin during the Oligocene-Miocene transition. Journal of Vertebrate Paleontology 31 (2): 479-496. https://doi.org/10.1080/02724634 .2011 .550360

Scherler L., Mennecart B., Hiard F. \& Becker D. 2013. Evolution of terrestrial hoofed-mammals during the Oligocene-Miocene transition in Europe. Swiss Journal of Geosciences 106: 349-369. https://doi.org/10.1007/s00015-013-0140-x

STEININGER F. 1999. - Chronostratigraphy, Geochronology and Biochronology of the Miocene "European Land Mammal Megazones" (ELMMZ) and the Miocene "Mammal-zones" (MNZ), in Rössner G. E. \& HeIssig K. (eds), The Miocene Land Mammals of Europe. F. Pfeil, München: 9-24.

TEMEY I. 1996. — Le Néogène de Touraine: approche environnementale et paléogéographique des faluns du bassin de NoyantSavigné (Indre-et-Loire et Maine-et-Loire, France). Mémoire d'Ingénieur géologue, Institut Géologique Albert-de-Lapparent, Cergy-Pontoise 73: 1-292, inédit.

YVARD J.-C. 1976. - Chronologie et sédimentation des calcaires lacustres du Sud-Ouest du Bassin de Paris dans leur environnement structural. Norois 92: 529-540. https://doi.org/10.3406/ noroi. 1976.3536 


\section{ANNEXES}

ANNEXE 1. - Tableau de corrélations stratigraphiques. Ce tableau présente les noms des âges mammaliens et locaux utilisés dans le texte par rapport aux âges géologiques standards.

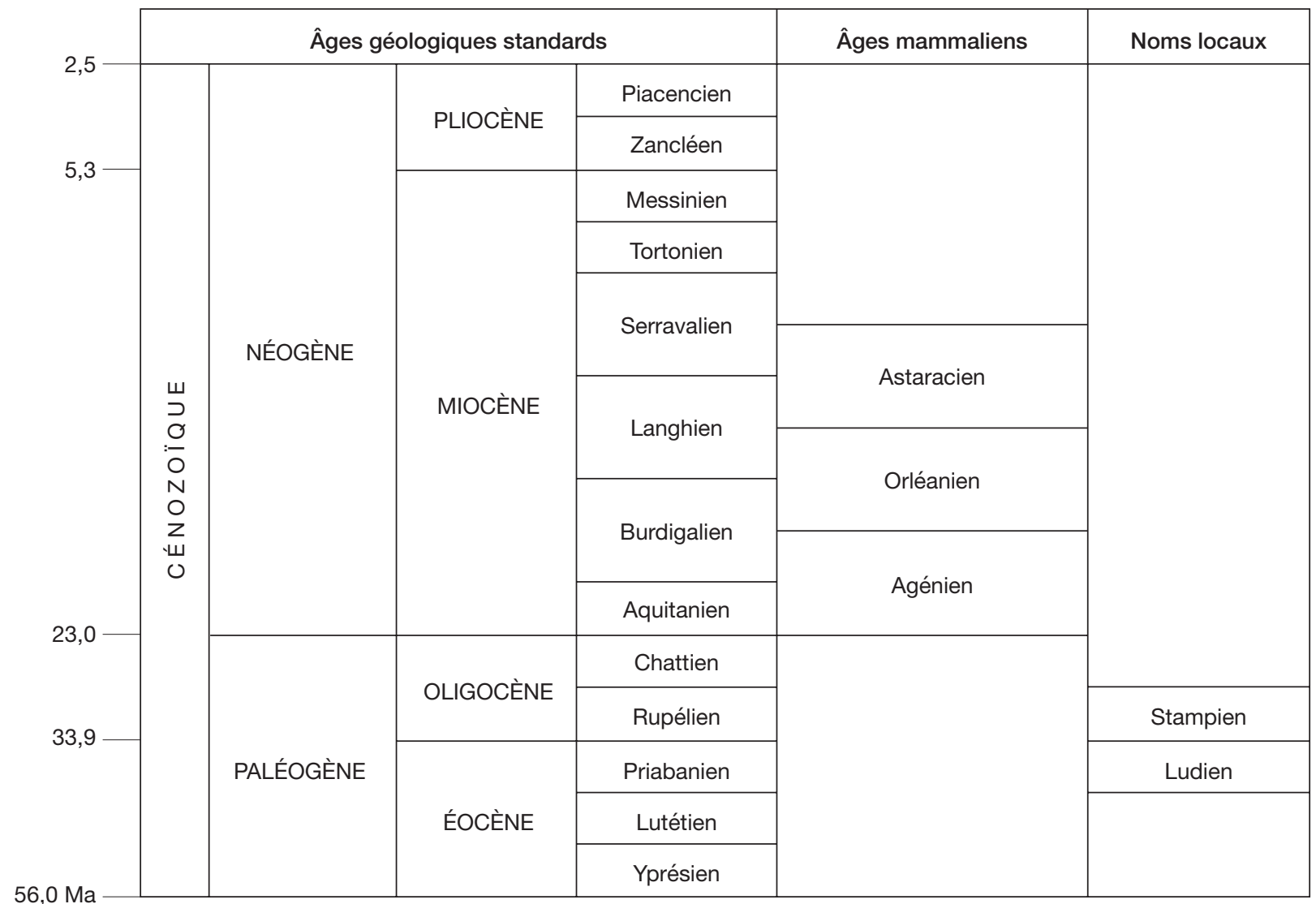

ANNEXE 2. - Liste des fossiles du Grand Morier consultés au musée du Savignéen.

Cette liste comprend 57 spécimens:

- Prodeinotherium bavaricum Von Meyer, 1831: P3 g. (2012. PBT.286), P3 g. (2012.PBT.287), P4 d. (2012.PBT.288), m1 d. (2012.PBT.285), M2 d. (2012.PBT.289);

- Paratapirus intermedius (Filhol, 1885): hémandibule d. portant p4-m1 (2012.PBT.2), hémimandibule d. portant m1-2 (2012.PBT.4), astragale g. (2012.PBT.347), astragale d. (2012.PBT.346);

- Brachypotherium brachypus (Lartet, 1837): i2 g. (2012. PBT.100), M3 g. (2012.PBT.102), métacarpien III g. (2012. PBT.208), métatarsien (2012.PBT.209);

- Diaceratherium aurelianense (Nouel, 1866): d3 d. (2012. PBT.57), d4 d. (2012.PBT.60), i2 g. (2012.PBT.40), p3 d. (2012.PBT.34), p4 g. (2012.PBT.31), P2 d. (2012.PBT.54), m1 g. (2012.PBT.27), M1 g. (2012.PBT.43), M2 g. (2012.
PBT.45), M3 g. (2012.PBT.51), M3 d. (2012.PBT.49), m2 g. (994-PBT-5).

- Protaceratherium minutum (Cuvier, 1822-1824): d2 d. (2012. PBT.145), d3 d. (2012.PBT.146), d4 d. (2012.PBT.305), I1 d. (2012.PBT.142), i2 g. (2012.PBT.124), montage d'une série dentaire portant P1-3 g. (2012.PBT.135), p2 g. (2012.PBT.113), p3 g. (2012.PBT.115), p4 g. (2012.PBT.121), p2 d. (2012. PBT.112), p3 d. (2012 .PBT.114), p4 d. (2012.PBT.118), P3 g. (2012.PBT.136), P4 g. (2012.PBT.139), P3 d. (2012.PBT.137), P4 d. (2012.PBT.140), m1 g. (2012.PBT.105), m1 d. (2012. PBT.104), m2 g. (2012.PBT.109), m2 d. (2012.PBT.108), m3 g. (2012.PBT.111), m3 d. (2012- PBT.110), M1 g. (2012. PBT.128), M2 d. (2012.PBT.130), M3 d. (2012-PBT.132).

- Prosantorhinus douvillei (Osborn, 1900): i2 g. (2012. PBT.157), i2 d. (2012.PBT.156), p2 g. (2012.PBT.153), p3 g. (2012.PBT.152), m3 d. (2012.PBT.148), M3 g. (2012.PBT.154). 
La liste présentée ci-après comporte 17 taxons de Chondrichthyens, sept taxons d'Osteichthyes, 14 taxons de Sauropsida dont une forme indéterminée d'oiseau et 64 taxons de mammiferres, soit un total de 102 taxons de vertébrés. Les taxons déjà reconnus par Ginsburg (2001) et Antunes \& Ginsburg (1989) sont marqués par le symbole «*".

\section{CLASSE CHONDRICHTHYENS HUXLEY, 1880}

Squalicorax sp.

Isurus desori (Agassiz, 1843)

Cosmopolitodus hastalis (Agassiz, 1843)

Otodus megalodon (Agassiz, 1837)

Carcharias (Odontaspis) acutissima (Agassiz, 1843)

ou Carcharias (Odontaspis) cuspidata (Agassiz, 1843)

Carcharias sp.

Cretolamna sp.

Carcharhinus priscus (Agassiz, 1843)

Galeocerdo aduncus (Agassiz, 1843)

Negaprion kraussi Probst, 1879

Hemipristis serra (Agassiz, 1835)

Notorhynchus primigenius (Agassiz, 1843)

Ptychodus sp.

Dasyatis sp.

Myliobatidae indet.

Aetobatus arcuatus (Agassiz, 1843)

Pristis aquitanicus Delfortrie, 1871

\section{SUPER-CLASSE OSTEICHTHYES HUXLEY, 1880}

Sparus cinctus (Agassiz, 1836)

Sparus aurata Linnaeus, 1758

Diplodus jomnitanus Valenciennes, 1844

Sphyraena olisiponensis Jonet, 1966

Labrodon pavimentatum (Gervais, 1857)

Tetrodon lecointrae Leriche, 1957

Trigonodon jugleri (Von Münster, 1846)

\section{CLASSE SAUROPSIDA HUXLEY, 1864}

Chelydropsis sp.

Ptychogaster sp.

Testudo promarginata Reinach, 1900

Testudo sp.

Trionyx sp.

Lacertidae indet.

Boidae indet.

Colubridae indet.

Viperidae indet.

Diplocynodon styriacus (Hofmann, 1885)

Diplocynodon sp.

Tomistoma cf. lusitanica (Vianna \& Morales, 1945)*

Gavialis sp.

Incertae sedis

\section{CLASSE MAMMALIA LINNAEUS, 1758}

Proscapanus sansaniensis (Lartet, 1851)

Lagopsis sp.

Eucricetodon infralactorensis (Viret, 1930)

Steneofiber depereti Mayet, 1908*

Anchitheriomys wiedemanni (Roger, 1885)*

Pliopithecus piveteaui Hürzeler, 1954*

Hyainailouros sulzeri Biedermann, 1863*

Amphicyon (Megamphicyon) lathanicus (Ginsburg, Chevenval, Janvier, Pouit \& Sen, 2000)*

Amphicyon giganteus (Schinz, 1825)*

Cynelos helbingi (Dehm, 1950)*

Cynelos schlosseri (Dehm, 1950)*

Agnotherium grivensis (Viret, 1929)*

Pseudocyon sansaniensis Lartet, 1851*

Pseudarctos bavaricus Schlosser, 1899*

Haplocyonoïdes mordax Hürzeler, 1940*

Ballusia hareni (Ginsburg, 1989)*

Phoberocyon aurelianensis (Frick, 1926)*

Phoberocyon hispanicus Ginsburg \& Morales, 1998*

Phoberocyon dehmi (Ginsburg, 1955)*

Plithocyon bruneti Ginsburg, 1980*

Hemicyon gargan Ginsburg \& Morales, 1998*

Hemicyon sansaniensis Lartet, 1851

Martes laevidens Dehm, 1950*

Trocharion albanense Major, 1903*

Protictitherium gaillardi Major, 1903*

Palaeogale hyaenoides (Dehm, 1950)*

Plesiogale angustifrons Pomel, 1854*

Pseudaelurus romievensis Viret, 1934*

Pseudaelurus lorteti Gaillard, 1899*

Pseudaelurus turnauensis Hoernes, 1882*

Prodeinotherium bavaricum Von Meyer, 1831* (Fig. 7)

Gomphotherium angustidens (Cuvier, 1817)*

Zygolophodon turicensis (Schinz, 1824)*

Metaxytherium sp.

Anchitherium aurelianense Von Meyer, 1844*

Anisodon grande (Lartet, 1851)*

Paratapirus intermedius (Filhol, 1885)* (Fig. 7)

Plagiolophus cf. annectens (Owen, 1847) (Fig. 7)

Ronzotherium cf. romani Kretzoi, 1945*

Brachypotherium brachypus (Lartet, 1837)*

Protaceratherium minutum (Cuvier, 1822-1824)* (Fig. 7)

Diaceratherium aurelianense (Nouel, 1866)* (Fig. 7)

Plesiaceratherium lumiarense Antunes \& Ginsburg, 1984*

Prosantorhinus germanicus (Wang, 1929)

Prosantorhinus douvillei (Osborn, 1900)*

Lartetotherium sansaniense (Lartet, 1851)* 
ANNEXE 3. - Suite.

Aureliachoerus aurelianensis (Stehlin, 1899)* Bunolistriodon lockarti (Pomel, 1848)* Hyotherium soemmeringi Von Meyer, 1841* Xenohyus venitor Ginsburg, 1980* Brachyodus onoideus (Gervais, 1869)* Brachyodus intermedius Mayet, 1908* Dorcatherium guntianum Von Meyer, 1847* Dorcatherium crassum Lartet, 1851* Amphimoschus pontileviensis Mayet, 1908*
Procervulus dichotomus Gervais, 1859*

Dicrocerus elegans Lartet, 1851*

Heterocemas sp.*

Lagomeryx sp.

Ligeromeryx praestans (Stehlin, 1937)*

Palaeomeryx bojani Von Meyer, 1834*

Andegameryx andegaviensis Ginsburg, 1971*

Cetacea indet.

Orycterocetus crocodilinus Cope, 1868 